\title{
Exchange Options under Clustered Jumps Dynamics
}

\author{
Yong Ma†, Dongtao Pan† and Tianyang Wang*‡ \\ $†$ College of Finance and Statistics, Hunan University, Changsha, 410079, China \\ $\ddagger$ College of Business, Colorado State University, Fort Collins, CO 80523, USA
}

(Received 00 Month 20XX; in final form 00 Month 20XX)

\begin{abstract}
Exchange options are one of the most popular exotic options, and have important implications for many common financial arrangements and for implied beta as a measure of systematic risk. In this study, we extend the existing literature on exchange options to allow for clustered jump contagion dynamics in each single asset, as well as across assets, using the Hawkes jump-diffusion model. We derive the analytical pricing formulae, the Greeks, and the optimal hedging strategy via Fourier transforms. Using an illustrative numerical analysis, we present the relationship between the exchange option price and clustered jump intensities and jump sizes in the underlying assets. We discuss the managerial insights on financial arrangements with exchange options characteristics. Furthermore, we discuss the implications of incorporating clustered jumps into the estimation of implied beta with exchange options, in which the applications can be insightful and useful in finance practice.
\end{abstract}

Keywords: Exchange Option; Hawkes Jump-Diffusion Process; Greeks; Optimal Hedging; Implied Beta

JEL Classification: C6, G13

\section{Introduction}

Exchange options offer the option holder the right, but not the obligation, to exchange one risky asset for another. First introduced by Margrabe (1978), and therefore also known as the Margrabe option, it is one of the earliest and the most popular exotic options in the over-the-counter (OTC) market.

Exchange options are designed to mitigate adverse relative movements across assets. Naturally, it is important for investors concerned with the relative performance of different assets and has become a widespread, effective risk mitigation tool in the stock, bond, commodity, and currency markets. Due to its generic nature, exchange options are related to many other derivatives. A vanilla European option on a single asset can be viewed as an option to exchange the asset with the same maturity zero-coupon bond at principal at the strike price (Merton 1973). Exchange options are also closely related to spread options, which derive their value from the difference, or spread, between the prices of two underlying assets against a strike price; the spread option reduces to an exchange option when the strike price is set to zero. Additionally, exchange options can be effectively combined with other types of derivative instruments. For example, an option on a swap (a.k.a., a swaption), which gives the holder the right to enter a swap at a later date to exchange one set of cash flows for another, is prevalent in OTC markets. Carr (1988) also discusses the sequential exchange opportunities as a compound exchange option when an exchange of assets creates the potential for future exchange.

${ }^{*}$ Corresponding author. Email: Tianyang.Wang@colostate.edu 
Moreover, many common financial arrangements are either equivalent to exchange options or have exchange options embedded. For instance, when a firm bids for another firm by offering its own stocks in exchange for the stocks of the target firm in mergers and acquisitions (M\&A), a common practice is to include a walk away covenant (i.e., a bringdown condition) in the exchange offer, which allows the bidder to walk away from the deal if the target firm's stock price drops significantly relative to that of the bidding firm. This walk away covenant is equivalent to an exchange option and can be valued accordingly. Similarly, a hedge fund may buy or sell OTC exchange options with the bidding and target firms' stocks as the underlying assets to either hedge or speculate on the risks of the M\&A falling apart due to a material deterioration in the target firm's business or financial condition between signing and closing. The embedded exchange option is also common in financial contracts. For instance, bondholders often have the right to convert their debt into equity. In addition, a portfolio manager's performance incentive fee arrangement, and margin account with collateral for the margin loan, are also important examples of exchange option-type arrangements in financial markets (Margrabe 1978; Rubinstein 1991). More generally, the basic idea of exchange options are applied to many fields, including real options (Trigeorgis 1993; Herath and Jahera Jr 2002), capital investment (Kensinger 1988), executive stock options (Johnson and Tian 2000), guaranteed funds (Hardy 2003; Biffis and Millossovich 2006), and insurance (Bühlmann 2004), among others.

In essence, the value of the exchange option depends not only on the current asset that might be exchanged and the life of the option, but also on the second asset and correlation between the two assets. In the case of a stock in relation to a market index, the exchange option price will incorporate important information about the option-implied correlations between the stock and the market. Therefore, exchange options have been proposed to estimate the systematic risk measured by beta (Siegel 1995), which is of central importance to both the theory and the practice of finance. Since the option market provides the investors' predictions about the future market movement, option-implied measurements have strong predictive power for the future volatility and higher moments (c.f. Corrado and Su 1997). Analogous to the concept of implied volatility, the exchange option-implied beta can reveal a unique, up-to-date market value for the beta of the stock with respect to the market index whenever such exchange options are traded ${ }^{1}$.

Given the rising importance of exchange options in the financial market, the increasing recognition of exchange option-type financial arrangements and the important implication of exchange option-implied beta for systematic risk, research on exchange options is of great value in finance for investors, practitioners, academics, and other market strategists. In this study, we join the extensive literature on exchange options. Since Margrabe (1978) first derived the closed-form expression for the European exchange option under the Black-Scholes framework, others extended this classic result under various extensions of the Black-Scholes model, such as the stochastic interest rate (Liu and Wang 1999), stochastic volatility (Antonelli et al. 2010), credit risk (Kim and Koo 2016), and the delayed underlying assets in the drift and diffusion parts (Lin et al. 2018). Since a prominent feature of financial markets is their infrequent, but large, price movements as jumps, recent studies showed considerable interest in evaluating exchange options with a jump-diffusion model that includes Poisson processes (Quittard-Pinon and Randrianarivony 2010), geometric compound Poisson jump processes (Cheang and Chiarella 2011), joint Poisson processes (Cufaro Petroni and Sabino 2018), Lévy processes (Chen and Wan 2010), the Ornstein-Uhlenbeck model (Pablo and Enrique 2017), the two-state Poisson capital asset pricing model (CAPM) (Kim et al. 2013), the fractional Black-Scholes model with jumps (Kim et al. 2014), and jumps with the possibility of default and a "bubble" in asset prices (Kardaras 2015).

\footnotetext{
${ }^{1}$ Since exotic options are mainly traded on the OTC market, exchange option prices are usually not available to the public (with the notable exception of some closely related spread options, e.g., exchange-traded WTI/Brent Crude Oil spread options in the Chicago Mercantile Exchange (CME), and energy spreads between the heating oil/crude oil and gasoline/crude oil crack in the New York Mercantile Exchange (NYMEX)). Siegel (1995) also briefly discusses synthetic exchange options according to the dynamic strategy of continuously adjusting the size invested in the underlying assets.
} 
Although the extant literature provides important insights on the valuation and hedging of exchange options, the strength of their potential implications is significantly mitigated, however, by a common and critical defect: prior studies virtually ignore the important phenomenon of clustered jumps, including jump clustering in a single asset and jump linkages between different assets. In reality, clustered jumps resulting from contagion or the propagation effect are regularly and repeatedly observed in the market (c.f. Maheu and McCurdy 2004; Ané and Métais 2011; Aït-Sahalia et al. 2015). Aït-Sahalia et al. (2015) argue that jumps can potentially influence future asset prices and can affect other assets due to macro news, investor attention, market sentiment, and other factors. The interplay between the various jump terms across markets and over time is not trivial and cannot be captured by the standard jump diffusion process. As the occurrence of a jump is likely to increase the probability and/or the magnitude of future jumps in both the asset itself and another risky asset (Luciano and Schoutens 2006), clustered jumps may have important implications in the valuation of exchange options and its hedging strategies. Additionally, this oversight may also have an important impact on the implication of the exchange option-implied beta, as clustered jumps could likely affect the measure of systematic risks.

While an exchange option is very simple by its design, it contains features at the source of some of the most acute problems in contemporary finance and offers a unique test bed to examine the foundations of stochastic asset pricing theory and the behavior of market participants under the dynamics of market contagion. In this study, we propose to value exchange option that allows for jump contagion in each single asset, as well as across assets, using a Hawkes jump-diffusion model. The Hawkes jump-diffusion model generalizes the usual Poisson jump-diffusion process (Hawkes 1971). In a Hawkes process, the jumps are interdependent, and the occurrence of a jump in the price of one asset increases the probability of jumps in its own price (i.e., univariate self-exciting processes), as well as the prices of other assets (i.e., mutually exciting processes). Consequently, Hawkes processes allow for both jump clustering in each asset and jump contagion across assets, and have wide applications in finance (c.f., Bacry and Muzy 2014; ?; Ma et al. 2017; ?; Hawkes 2018). To account for the behaviors observed in the financial market, prior studies propose mutually exciting Hawkes processes to model clustered jumps, such as shocks to the credit default swap (CDS) markets (Aït-Sahalia et al. 2015), microstructure noise (Bacry et al. 2013), and structural credit risk (Ma and Xu 2016), among others. For instance, Aït-Sahalia et al. (2015) propose capturing the contagious dynamics of asset returns through both mutually self-exciting and cross-exciting clustered Hawkes jumps in the context of international stock index returns for five world regions. Kokholm (2016) studies the contagion effect on multi-asset derivatives and calculates the hedge ratios for European put and call options. Additionally, others propose self-exciting Hawkes models to model joint defaults in the portfolios of credit derivatives (Errais et al. 2006). This recent stream of literature provides significant evidence of clustered jumps, both in the asset and the derivatives markets, especially during large and volatile movements in the markets. They also indicate the critical importance of extending the existing asset pricing and derivatives models by incorporating dynamic clustered jumps due to the complex interplay of clustered jumps, both across assets and over time.

Although the clustered jump modeled by Hawkes processes may have natural and important implications for exchange options valuation and hedging, to the best of our knowledge, its use has not been studied or explored in the literature. In the same spirit of this new stream of literature, this study contributes to the development and implementation of valuation, hedging, and testing procedures for exchange options. Moreover, we also further the discussion on the economic implications of clustered jumps in the context of the implied beta and financial arrangements with exchange options characteristics. More specifically, our research integrates work on exchange option pricing with research on the Hawkes jump-diffusion model and the implied beta. We contribute to the existing literature in at least three important ways. First, we develop a new method to derive the closed-form pricing formula for European exchange options under the Hawkes jump-diffusion model. The presence of clustered jumps makes it very challenging to implement because it can quickly become mathematically non-treatable. We successfully address this problem via the Fourier 
transform technique. Second, our model accounts for the interacting dynamics of the underlying assets jumps, and we thus obtain the exchange option's Greeks and derive the optimal dynamic hedging strategy under the mean-variance criterion. Additionally, we demonstrate the sensitivity of the exchange option price to the parameters related to Hawkes jumps. Third, we discuss the important implications of incorporating clustered jumps into the exchange option-implied beta and compare it with other models. Our study yields several findings. First, the exchange option value increases with jump volatility, the absolute value of mean jump size, the intrinsic intensity and intensity jump size, and decreases with exponential decay rate. The fluctuation of the underlying assets could account for the effects of the parameters related to jumps on the option value because the occurrence of jumps and the jump amplitude increase the fluctuation of the underlying assets, which in turn contributes to the option value. Second, the effects of two underlying assets on the value of the exchange option are symmetric, and the effects of positive and negative mean jump sizes on the exchange option price are asymmetric. Third, ignoring jumps and clustered jumps will significantly misestimate the implied beta, and these errors may carry forward to important issues such as estimating the cost of capital, measuring performance, and detecting abnormal returns.

The remainder of this paper is organized as follows. In Section 2, we introduce the Hawkes jump-diffusion process and derive its joint characteristic function under the general affine jumpdiffusion framework. In Section 3, we obtain the European exchange option price by incorporating clustered jumps using the Fourier transform method. In Section 4, we present the Greeks, the optimal hedging strategies, and sensitivity of the option prices to the parameters of Hawkes jump intensities and jump sizes. We also discuss the managerial insights on financial arrangements with the characteristics of exchange options. In Section 5, we discuss the implied beta under clustered jumps and its economic implications, and provide a numerical investigation its estimation under different models. In Section 6, we propose a hybrid approach and implement an empirical analysis to examine its forecasting performance for the implied beta. Section 7 concludes this paper.

\section{The Hawkes Model}

To capture the interacting dynamics among different jumps and across different assets as a means to evaluate their impacts on exchange option values properly, we propose a model based on mutually exciting Hawkes jump-diffusion processes with the characteristics of both self-excitement and crossexcitement. In this section, we first provide a brief overview of mutually exciting point processes and then specify our proposed model in detail.

\subsection{Hawkes Jump-diffusion Processes}

There are two underlying assets in an exchange option, with prices of $S_{j, t}$ for asset $j$ at time $t$, $j=1,2$. Consider a filtered probability space $\left(\Omega, \mathcal{F},\left\{\mathcal{F}_{t}\right\}_{t \geq 0}, \mathbb{Q}\right)$, where $\left\{\mathcal{F}_{t}\right\}$ is a right-continuous and complete filtration representing the available information set and $\mathbb{Q}$ is a risk-neutral probability measure. We can model the underlying asset prices as

$$
S_{j, t}=S_{j, 0} e^{\int_{0}^{t} \mu_{j, s} d s+X_{j, t}}, \quad j=1,2,
$$

where $\mu_{j, s}$ are the expected rates of returns on the underlying assets at time $s$. Following Kokholm (2016), the processes $X_{j, t}$ have the initial conditions $X_{j, 0}=0$ and follow these dynamics:

$$
d X_{j, t}=\left(-\frac{1}{2} \sigma_{j, t}^{2}-\xi_{j} \lambda_{j, t}\right) d t+\sigma_{j, t} d W_{j, t}+d J_{j, t},
$$

where $\sigma_{j, t}$ are the volatilities of the underlying assets at time $t, W_{j, t}$ are standard Brownian motions independent of the pure jump processes $J_{j, t}$ with intensities $\lambda_{j, t}$, and $d W_{1, t} d W_{2, t}=\rho_{t} d t$. Further- 
more, let $\tau_{j, l}$ denote the time of occurrence of the $l$-th jump in asset $j$ and $N_{j, t}=\sum_{l=1}^{\infty} \mathbf{1}\left\{\tau_{j, l} \leq t\right\}$ is the cumulant of the jumps in asset $j$ up to time $t$. Let $d J_{j, t}=\varepsilon_{j, t} d N_{j, t}$, where $\varepsilon_{j, t}$ are random jump sizes with distribution functions of $F_{j}$ across $t$ and are independent of each other for all $t$ and $j . \xi_{j}=\int_{-\infty}^{\infty}\left(e^{y}-1\right) d F_{j}(y)$ are the mean price-jump sizes. Then, the drifts in (2) can ensure that $e^{X_{j, t}}$ are martingales under $\mathbb{Q}$. Assume that $N_{i, t}, i=1,2$, are mutually exciting Hawkes processes with intensity processes $\lambda_{j, t}$ given by

$$
d \lambda_{j, t}=\eta_{j}\left(\lambda_{j, \infty}-\lambda_{j, t}\right) d t+\sum_{l=1}^{2} \theta_{j, l} d N_{l, t}
$$

or

$$
d \lambda_{j, t}=\eta_{j}\left(\lambda_{j, \infty}-\lambda_{j, t}\right) d t+\sum_{l=1}^{2} \theta_{j, l} d J_{l, t}
$$

where $\eta_{j}, \lambda_{j, \infty}$ and $\theta_{j, l}$ are positive constants. If we use (4) to specify the intensity, then the supports of $F_{l}$ must be subsets of $(0, \infty)$ (e.g., lognormal or exponential distribution) to guarantee that the intensities are always positive. Under either specification, when a jump occurs in one process, the jump intensities of both processes will increase instantly, but the impact of this jump will decay exponentially over time. $\lambda_{j, \infty}, \theta_{j, l}$, and $\eta_{j}$ reflect the intrinsic jump intensities, the self-excitement or cross-excitement effects, and decay rates, respectively. The main distinction between (3) and (4) is that for the former, the increment of intensity at any jump is constant, whereas for the latter, it also depends on the jump size. In particular, if $\theta_{j, l}=\eta_{j, l}=0$, then $X_{j, t}$ degenerate to Poisson jump-diffusion processes.

\subsection{Characteristic Functions of Hawkes Processes}

In the following, we derive the characteristic functions of the Hawkes jump diffusion processes. Let $X_{t}=\left(X_{1, t}, X_{2, t}\right)^{\prime}$ and $u=\left(u_{1}, u_{2}\right)^{\prime}$; then, with $\mathbb{E}$ denoting the expectation under the probability measure $\mathbb{Q}$, the time- $t$ conditional joint characteristic function for the process $X_{t}$ at the future time $T$ is

$$
\Phi\left(u_{1}, u_{2}, t, T\right)=\mathbb{E}\left[e^{i u^{\prime} X_{T}} \mid \mathcal{F}_{t}\right],
$$

where $i$ is the imaginary unit; that is, $i=\sqrt{-1}$.

We can express the marginal condition characteristic functions for $X_{1}$ and $X_{2}$ as

$$
\left\{\begin{array}{l}
\Phi_{1}\left(u_{1}, t, T\right):=\mathbb{E}\left[e^{i u_{1} X_{1, T}} \mid \mathcal{F}_{t}\right]=\Phi\left(u_{1}, 0, t, T\right), \\
\Phi_{2}\left(u_{2}, t, T\right):=\mathbb{E}\left[e^{i u_{2} X_{2, T}} \mid \mathcal{F}_{t}\right]=\Phi\left(0, u_{2}, t, T\right) .
\end{array}\right.
$$

We can easily decompose $X_{t}$ into $X_{t}=X_{t}^{C}+X_{t}^{J}$ by defining

$$
X_{t}^{C}=\left(-\frac{1}{2} \int_{0}^{t} \sigma_{1, s}^{2} d s+\int_{0}^{t} \sigma_{1, s} d W_{1, s}, \quad-\frac{1}{2} \int_{0}^{t} \sigma_{2, s}^{2} d s+\int_{0}^{t} \sigma_{2, s} d W_{2, s}\right)^{\prime},
$$

and

$$
X_{t}^{J}=\left(-\xi_{1} \int_{0}^{t} \lambda_{1, s} d s+J_{1, t}, \quad-\xi_{2} \int_{0}^{t} \lambda_{2, s} d s+J_{2, t}\right)^{\prime}
$$


Moreover, since $W_{t}$ and $J_{t}$ are independent, we have

$$
\Phi\left(u_{1}, u_{2}, t, T\right)=\Phi_{C}\left(u_{1}, u_{2}, t, T\right) \Phi_{J}\left(u_{1}, u_{2}, t, T\right),
$$

where $\left.\Phi_{C}\left(u_{1}, u_{2}, t, T\right)=\mathbb{E}\left[e^{i u^{\prime} X_{T}^{C}} \mid \mathcal{F}_{t}\right]\right)$ and $\Phi_{J}\left(u_{1}, u_{2}, t, T\right)=\mathbb{E}\left[e^{i u^{\prime} X_{T}^{J}} \mid \mathcal{F}_{t}\right]$. Next, we show how we calculate $\Phi_{C}\left(u_{1}, u_{2}, t, T\right)$ and $\Phi_{J}\left(u_{1}, u_{2}, t, T\right)$.

It is obvious that $X_{T}^{C}-X_{t}^{C}$ follows a bivariate normal distribution with mean $m_{t, T}:=$ $\left(-\frac{1}{2} \int_{t}^{T} \sigma_{1, s}^{2} d s,-\frac{1}{2} \int_{t}^{T} \sigma_{2, s}^{2} d s\right)^{\prime}$ and covariance matrix

$$
c_{t, T}:=\left[\begin{array}{cc}
\int_{t}^{T} \sigma_{1, s}^{2} d s & \int_{t}^{T} \rho_{s} \sigma_{1, s} \sigma_{2, s} d s \\
\int_{t}^{T} \rho_{s} \sigma_{1, s} \sigma_{2, s} d s & \int_{t}^{T} \sigma_{2, s}^{2} d s
\end{array}\right] .
$$

Hence,

$$
\Phi_{C}\left(u_{1}, u_{2}, t, T\right)=e^{i u^{\prime} X_{t}^{C}} \mathbb{E}\left[e^{i u^{\prime}\left(X_{T}^{C}-X_{t}^{C}\right)} \mid \mathcal{F}_{t}\right]=e^{i u^{\prime}\left(X_{t}^{C}+m_{t, T}\right)-\frac{1}{2} u^{\prime} c_{t, T} u}
$$

On the other hand, we can derive $\Phi_{J}\left(u_{1}, u_{2}, t, T\right)$ under the general framework of affine jump diffusion models. $\mathbf{0}_{V}$ denotes a bi-dimensional row vector of zeros, $\mathbf{0}_{M}$ a $2 \times 2$ matrix of zeros, and similarly, $\mathbf{1}_{V}$ a bi-dimensional row vector of ones. $\mathbf{1}_{j}$ a bi-dimensional row vector with one at the $j$-th entry and zero at the other entry. According to Errais et al. (2006) and Kokholm (2016),

$$
Y_{t}=\left(\lambda_{1, t}, \lambda_{2, t},-\xi_{1} \int_{0}^{t} \lambda_{1, s} d s+J_{1, t},-\xi_{2} \int_{0}^{t} \lambda_{2, s} d s+J_{2, t}\right)^{\prime}
$$

is a 4-dimensional affine jump-diffusion process satisfying

$$
d Y_{t}=\left(\alpha+\beta Y_{t}\right) d t+\sum_{j=1}^{2} \gamma_{j} d L_{j, t},
$$

and

$$
\lambda_{j, t}=\delta_{j} Y_{t}
$$

where $\alpha=\left(\eta_{1} \lambda_{1, \infty}, \eta_{2} \lambda_{2, \infty}, \mathbf{0}_{V}\right)^{\prime}$ and

$$
\beta=\left[\begin{array}{ll}
\operatorname{diag}\left(-\eta_{1},-\eta_{2}\right) & \mathbf{0}_{M} \\
\operatorname{diag}\left(-\xi_{1},-\xi_{2}\right) & \mathbf{0}_{M}
\end{array}\right]
$$

In addition, for each $j, \gamma_{j}=\operatorname{diag}\left(\theta_{1, j}, \theta_{2, j}, \mathbf{1}_{j}\right), \delta_{j}=\left(\mathbf{1}_{j}, \mathbf{0}_{V}\right)$, and $L_{j, t}$ is a 4-dimensional pure jump process in which $L_{j, t}=\left(N_{j, t} \mathbf{1}_{V}, J_{j, t} \mathbf{1}_{V}\right)^{\prime}$ for (3) and $L_{j, t}=\left(J_{j, t} \mathbf{1}_{V}, J_{j, t} \mathbf{1}_{V}\right)^{\prime}$ for (4). According to Duffie et al. (2000), we can obtain the time- $t$ conditional characteristic function of $Y_{t}$ at the future time $T$ by

$$
\Phi_{Y}(\nu, t, T)=\mathbb{E}\left[e^{\nu^{\prime} Y_{T}} \mid \mathcal{F}_{t}\right]=e^{A(\nu, t, T)+B(\nu, t, T)^{\prime} Y_{t}}, \quad \nu \in \mathbb{C}^{4}
$$

where $A(\nu, t, T)$ and $B(\nu, t, T)=\left(B_{1}(\nu, t, T), \ldots, B_{4}(\nu, t, T)\right)^{\prime}$ satisfy the ordinary differential equa- 
tions (ODEs):

$$
\left\{\begin{array}{l}
\frac{\partial A}{\partial t}=-\alpha^{\prime} B \\
\frac{\partial B}{\partial t}=-\beta^{\prime} B-\sum_{j=1}^{2}\left(\Psi_{j}\left(\gamma_{j} B\right)-1\right) \delta_{j}^{\prime}
\end{array}\right.
$$

with boundary conditions $A(\nu, T, T)=0$ and $B(\nu, T, T)=\nu$. In addition, we obtain $\Psi_{j}$ using

$$
\Psi_{j}(\omega)=\int_{\mathbb{R}} e^{\omega^{\prime} \varphi} d F_{j}(y), \omega \in \mathbb{C}^{4}
$$

where $\varphi=\left(\mathbf{1}_{V}, y \mathbf{1}_{V}\right)^{\prime}$ for (3) and $\varphi=\left(y \mathbf{1}_{V}, y \mathbf{1}_{V}\right)^{\prime}$ for (4). Consequently, we have

$$
\Phi_{J}\left(u_{1}, u_{2}, t, T\right)=\Phi_{Y}\left(\left(\mathbf{0}_{V}, i u_{1}, i u_{2}\right)^{\prime}, t, T\right) .
$$

In general, there are no closed-form solutions for $A(\nu, t, T)$ and $B(\nu, t, T)$. However, if we reduce the Hawkes processes to Poisson processes, then the closed-form solutions are available. In this study, we will solve the ODEs numerically using the Runge-Kutta algorithm (Butcher 1987).

\section{Pricing Exchange Options with Hawkes Processes}

The traditional methods of pricing exchange options by directly solving a partial differential equation (PDE) are limited under the Hawkes clustered jumps processes. In this section, we propose a new approach to price the exchange option that incorporates clustered jumps via the Fourier transform technique.

\subsection{Change in the Measure and Risk-neutral Pricing}

An exchange option gives the holder the right to exchange asset two for asset one at expiration time $T$. Its payoff at time $T$ is $V_{T}=\max \left\{S_{1, T}-S_{2, T}, 0\right\}=\left(S_{1, T}-S_{2, T}\right)^{+}$. According to the risk-neutral pricing approach, the value of the exchange option at time $0 \leq t \leq T$ is

$$
V_{t}=e^{-r(T-t)} \mathbb{E}\left[\left(S_{1, T}-S_{2, T}\right)^{+} \mid \mathcal{F}_{t}\right],
$$

where $r$ is the risk-free interest rate. For simplicity of exposition, we assume $\mu_{j, t}=\mu_{j}, \sigma_{j, t}=\sigma_{j}$, and $\rho_{t}=\rho, j=1,2$ in this study, but the framework extends easily to the more general cases. Since $e^{\left(q_{j}-r\right) t} S_{j, t}$ are martingales under the risk-neutral measure $\mathbb{Q}$, it is obvious that $\mu_{j}=r-q_{j}$, where $q_{j}$ is the continuously paying dividend yield of asset $j$.

We define another probability measure $\widetilde{\mathbb{Q}}$ by $\frac{d \widetilde{\mathbb{Q}}}{d \mathbb{Q}}=e^{X_{2, T}}$; that is, $e^{X_{2, T}}$ is the Radon-Nikodým derivative of $\widetilde{\mathbb{Q}}$ with respect to $\mathbb{Q}$. Let $\widetilde{\mathbb{E}}$ be the expectation under the probability measure $\widetilde{\mathbb{Q}}$; then, we can rewrite the value of the exchange option at time $t$ as

$$
V_{t}=e^{-r(T-t)} \mathbb{E}\left[S_{2, T}\left(\frac{S_{1, T}}{S_{2, T}}-1\right)^{+} \mid \mathcal{F}_{t}\right]=S_{2, t} e^{-q_{2}(T-t)} \widetilde{\mathbb{E}}\left[\left(\frac{S_{1, T}}{S_{2, T}}-1\right)^{+} \mid \mathcal{F}_{t}\right] .
$$

Furthermore, let $\widetilde{\Phi}\left(u_{1}, u_{2}, t, T\right)$ denote the time-t-conditional joint characteristic function for $X_{t}$ at 
future time $T$ under the probability measure $\widetilde{\mathbb{Q}}$; then, we have

$$
\widetilde{\Phi}\left(u_{1}, u_{2}, t, T\right)=\widetilde{\mathbb{E}}\left[e^{i u_{1} X_{1, T}+i u_{2} X_{2, T}} \mid \mathcal{F}_{t}\right]=e^{-X_{2, t}} \Phi\left(u_{1}, u_{2}-i, t, T\right) .
$$

\subsection{The Fourier Transform of Exchange Option Prices}

In this subsection, we use a Fourier transform to derive the value of the exchange option. Let

$$
K\left(u_{1}, u_{2}, t, T\right)=\frac{1}{u_{1} u_{2}} \exp \left\{\sum_{j=1}^{3} K_{j}\left(u_{1}, u_{2}, t, T\right)\right\}
$$

and

$$
\left\{\begin{array}{l}
K_{1}\left(u_{1}, u_{2}, t, T\right)=\sum_{j=1}^{2} u_{j}\left[\log S_{j, t}-q_{j}(T-t)\right] \\
K_{2}\left(u_{1}, u_{2}, t, T\right)=-\frac{1}{2} u_{1} u_{2} \sigma^{2}(T-t) \\
K_{3}\left(u_{1}, u_{2}, t, T\right)=A^{*}\left(u_{1}, u_{2}, t, T\right)+\sum_{j=1}^{2} B_{j}^{*}\left(u_{1}, u_{2}, t, T\right) \lambda_{j, t}
\end{array}\right.
$$

where $\sigma=\sqrt{\sigma_{1}^{2}+\sigma_{2}^{2}-2 \rho \sigma_{1} \sigma_{2}}$, and

$$
\left\{\begin{array}{l}
A^{*}\left(u_{1}, u_{2}, t, T\right)=A\left(\left(\mathbf{0}_{V}, u_{1}, u_{2}\right)^{\prime}, t, T\right), \\
B_{j}^{*}\left(u_{1}, u_{2}, t, T\right)=B_{j}\left(\left(\mathbf{0}_{V}, u_{1}, u_{2}\right)^{\prime}, t, T\right)
\end{array}\right.
$$

with $A\left(\left(\mathbf{0}_{V}, u_{1}, u_{2}\right)^{\prime}, t, T\right)$ and $B_{j}\left(\left(\mathbf{0}_{V}, u_{1}, u_{2}\right)^{\prime}, t, T\right)$ obtained from the ODEs (10).

THEOREM 3.1 We can write the exchange option value under a clustered jump process as

$$
V_{t}=S_{1, t} e^{-q_{1}(T-t)}-\frac{1}{\pi} \int_{0}^{\infty} \operatorname{Re}\left[K\left(\frac{1}{2}+i w, \frac{1}{2}-i w, t, T\right)\right] d w
$$

where $\operatorname{Re}(\cdot)$ is the real part of a complex number.

Proof. Let $k_{1}=\log S_{1,0}-\log S_{2,0}+\left(\mu_{1}-\mu_{2}\right) T$ and $k_{2}=\log S_{1,0}+\log S_{2,0}+\left(\mu_{1}+\mu_{2}\right) T$. The generalized Fourier transform of $G(x)=\left(e^{k_{1}+x}-1\right)^{+}$is

$$
\widehat{G}(z)=\int_{-\infty}^{\infty} e^{i z x} G(x) d x=-\frac{e^{-i z k_{1}}}{z^{2}-i z}, \quad z \in \mathbb{C}, \quad \operatorname{Im}(z)>1,
$$

where $\operatorname{Im}(\cdot)$ is the imaginary part of a complex number. The generalized Fourier inverse transform of $G(x)$ is

$$
G(x)=\frac{1}{2 \pi} \int_{i v-\infty}^{i v+\infty} e^{-i z x} \widehat{G}(z) d z, \quad v=\operatorname{Im}(z) .
$$


From (13), (14), and (17), it follows that the integral expression for the exchange option value is

$$
\begin{aligned}
V_{t} & =S_{2, t} e^{-q_{2}(T-t)} \widetilde{\mathbb{E}}\left[G\left(X_{1, T}-X_{2, T}\right) \mid \mathcal{F}_{t}\right] \\
& =\frac{S_{2, t} e^{-q_{2}(T-t)}}{2 \pi} \widetilde{\mathbb{E}}\left[\int_{i v-\infty}^{i v+\infty} e^{-i z\left(X_{1, T}-X_{2, T}\right)} \widehat{G}(z) d z \mid \mathcal{F}_{t}\right] \\
& =\frac{S_{2, t} e^{-q_{2}(T-t)}}{2 \pi} \int_{i v-\infty}^{i v+\infty} \widetilde{\mathbb{E}}\left[e^{-i z\left(X_{1, T}-X_{2, T}\right)} \mid \mathcal{F}_{t}\right] \widehat{G}(z) d z \\
& =\frac{S_{2,0} e^{r t-q_{2} T}}{2 \pi} \int_{i v-\infty}^{i v+\infty} \Phi(-z, z-i, t, T) \widehat{G}(z) d z .
\end{aligned}
$$

Moreover, we can change the integration contour into $\operatorname{Im}(z) \in(0,1)$ by the residual theorem (c.f., Lewis 2001). Let $v=\frac{1}{2}$ and $z=w+\frac{1}{2} i$; then,

$$
\begin{aligned}
V_{t} & =\frac{S_{2,0} e^{r t-q_{2} T}}{2 \pi}\left[2 \pi e^{k_{1}} \Phi(-i, 0, t, T)-\int_{-\infty}^{\infty} \frac{e^{\left(\frac{1}{2}-i w\right) k_{1}} \Phi\left(-w-\frac{i}{2}, w-\frac{i}{2}, t, T\right)}{w^{2}+1 / 4} d w\right] \\
& =S_{1, t} e^{-q_{1}(T-t)}-\frac{e^{\frac{1}{2} k_{2}-r(T-t)}}{\pi} \int_{0}^{\infty} \frac{\operatorname{Re}\left[e^{i w k_{1}} \Phi\left(w-\frac{i}{2},-w-\frac{i}{2}, t, T\right)\right]}{w^{2}+1 / 4} d w
\end{aligned}
$$

By simple calculation, we have

$$
V_{t}=S_{1, t} e^{-q_{1}(T-t)}-\frac{1}{\pi} \int_{0}^{\infty} \operatorname{Re}\left[K\left(\frac{1}{2}+i w, \frac{1}{2}-i w, t, T\right)\right] d w
$$

Note that since $V_{t}=e^{-r(T-t)} \mathbb{E}\left[\left(S_{1, T}-S_{2, T}\right)^{+} \mid \mathcal{F}_{t}\right]<e^{-r(T-t)} \mathbb{E}\left[S_{1, T} \mid \mathcal{F}_{t}\right]=S_{1, t} e^{-q_{1}(T-t)}$, it follows that

$$
\int_{0}^{\infty} \operatorname{Re}\left[K\left(\frac{1}{2}+i w, \frac{1}{2}-i w, t, T\right)\right] d w>0
$$

\section{Greeks and Optimal Hedging Strategy}

In this section, we discuss the optimal hedging strategy for exchange options under clustered jumps, and present the Greeks for the corresponding exchange option, including Delta, Theta, Vega, Gamma, and other partial derivatives. Then, we illustrate the effects of Hawkes-type jumps with numerical examples on exchange option prices.

\section{1. $\quad$ The Optimal Hedging Strategy}

Since jumps are present in the proposed model, perfect hedges for exchange options do not exist. Here, we apply the mean-variance hedging (quadratic hedging) approach to obtain an optimal hedging strategy. In a mean-variance hedging, we describe the optimal self-financing strategy by an initial capital of $P_{0}$ and a portfolio process $\Delta_{t}=\left(\Delta_{1, t}, \Delta_{2, t}\right)$, where $\Delta_{j, t}$ is the number of shares of asset $j$ at time $t$. The objective is to minimize the terminal hedging error in the mean-variance sense:

$$
\inf _{\Delta_{t}} \mathbb{E}\left(P_{T}-V_{T}\right)^{2}
$$


or equivalently, $\inf _{\Delta_{t}} \mathbb{E}\left[\left(e^{-r T} P_{T}-e^{-r T} V_{T}\right)^{2}\right]$, where the hedge portfolio value $P_{t}$ is driven by

$$
d P_{t}=\sum_{j=1}^{2}\left[\Delta_{j, t}\left(q_{j} S_{j, t}+d S_{j, t}\right)\right]+r\left(P_{t}-\sum_{j=1}^{2} \Delta_{j, t} S_{j, t}\right) d t
$$

Let $V\left(t, s_{1}, s_{2}, \lambda_{1}, \lambda_{2}\right)$ denote the value of the exchange option at time $t$ if the underlying asset prices at that time are $S_{j, t}=s_{j}$ and the intensities are $\lambda_{j, t}=\lambda_{j}, j=1,2$. Additionally, let

$$
V_{j, t}^{S}=\frac{\partial V}{\partial s_{j}}\left(t, S_{1, t}, S_{2, t}, \lambda_{1, t}, \lambda_{2, t}\right), \quad j=1,2
$$

Furthermore, if the intensity processes $\lambda_{j, t}$ are as in (3), then let

$$
\left\{\begin{array}{l}
V_{1, t, y}^{J}=V\left(t, S_{1, t^{-}} e^{y}, S_{2, t}, \lambda_{1, t^{-}}+\theta_{11}, \lambda_{2, t^{-}}+\theta_{21}\right)-V\left(t, S_{1, t^{-}}, S_{2, t}, \lambda_{1, t^{-}}, \lambda_{2, t^{-}}\right), \\
V_{2, t, y}^{J}=V\left(t, S_{1, t}, S_{2, t^{-}} e^{y}, \lambda_{1, t^{-}}+\theta_{12}, \lambda_{2, t^{-}}+\theta_{22}\right)-V\left(t, S_{1, t}, S_{2, t^{-}}, \lambda_{1, t^{-}}, \lambda_{2, t^{-}}\right)
\end{array}\right.
$$

and if $\lambda_{j, t}$ are given by (4), then let

$$
\left\{\begin{array}{l}
V_{1, t, y}^{J}=V\left(t, S_{1, t^{-}} e^{y}, S_{2, t}, \lambda_{1, t^{-}}+\theta_{11} y, \lambda_{2, t^{-}}+\theta_{21} y\right)-V\left(t, S_{1, t^{-}}, S_{2, t}, \lambda_{1, t^{-}}, \lambda_{2, t^{-}}\right), \\
V_{2, t, y}^{J}=V\left(t, S_{1, t}, S_{2, t^{-}} e^{y}, \lambda_{1, t^{-}}+\theta_{12} y, \lambda_{2, t^{-}}+\theta_{22} y\right)-V\left(t, S_{1, t}, S_{2, t^{-}}, \lambda_{1, t^{-}}, \lambda_{2, t^{-}}\right) .
\end{array}\right.
$$

TheOREM 4.1 Let $\zeta_{j}=\int_{-\infty}^{\infty}\left(e^{y}-1\right)^{2} d F_{j}(y)$ and $\phi_{j, t}=\int_{-\infty}^{\infty}\left(e^{y}-1\right) V_{j, t, y}^{J} d F_{j}(y)$. The optimal hedging portfolio is

$$
\hat{\Delta}_{t}=\left(\hat{\Delta}_{1, t}, \hat{\Delta}_{2, t}\right)^{\prime}=\underset{\Delta_{t}}{\operatorname{argmin}} \mathbb{E} \epsilon_{T}^{2}=a_{t}^{-1} b_{t}
$$

where

$$
a_{t}=\left(\begin{array}{cc}
\left(\sigma_{1}^{2}+\lambda_{1, t} \zeta_{1}\right) S_{1, t} & \rho \sigma_{1} \sigma_{2} S_{2, t} \\
\rho \sigma_{1} \sigma_{2} S_{1, t} & \left(\sigma_{2}^{2}+\lambda_{2, t} \zeta_{2}\right) S_{2, t}
\end{array}\right)
$$

and

$$
b_{t}=\left(\begin{array}{l}
\sigma_{1}^{2} S_{1, t} V_{1, t}^{S}+\rho \sigma_{1} \sigma_{2} S_{2, t} V_{2, t}^{S}+\lambda_{1, t} \phi_{1, t} \\
\sigma_{2}^{2} S_{2, t} V_{2, t}^{S}+\rho \sigma_{1} \sigma_{2} S_{1, t} V_{1, t}^{S}+\lambda_{2, t} \phi_{2, t}
\end{array}\right)
$$

Proof. Obviously, we have

$$
d\left(e^{-r t} P_{t}\right)=e^{-r t} \sum_{j=1}^{2}\left(\sigma_{j} S_{j, t} \Delta_{j, t} d W_{j, t}+S_{j, t} \Delta_{j, t} d J_{j, t}^{*}\right)
$$

where

$$
d J_{j, t}^{*}=\left(e^{\varepsilon_{j, t}}-1\right) d N_{j, t}-\xi_{j} \lambda_{j, t} d t, j=1,2
$$


Since $e^{-r t} V_{t}$ is a martingale under the risk-neutral probability measure $\mathbb{Q}$,

$$
d\left(e^{-r t} V_{t}\right)=e^{-r t} \sum_{j=1}^{2}\left(\sigma_{j} S_{j, t} V_{j, t}^{S} d W_{j, t}+V_{j, t, \varepsilon_{j, t}}^{J} d N_{j, t}^{*}\right),
$$

where $d N_{j, t}^{*}=d N_{j, t}-\lambda_{j, t} d t$. Let

$$
\epsilon_{t}=e^{-r t}\left(P_{t}-V_{t}\right), \quad \epsilon_{j, t}^{W}=e^{-r t} \sigma_{j} S_{j, t}\left(\Delta_{j, t}-V_{j, t}^{S}\right), \quad \epsilon_{j, t}^{J}=e^{-r t} S_{j, t} \Delta_{j, t}, \quad \epsilon_{j, t, y}^{N}=e^{-r t} V_{j, t, y}^{J},
$$

and $\epsilon_{j, t, y}^{J}=\left(e^{y}-1\right) \epsilon_{j, t}^{J}-\epsilon_{j, t, y}^{N}, j=1,2$. According to Itô's formula for a semi-martingale,

$$
\begin{aligned}
\epsilon_{T}^{2}= & \int_{0}^{T}\left\{2 \rho \epsilon_{1, t}^{W} \epsilon_{2, t}^{W}+\sum_{j=1}^{2}\left[\left(\epsilon_{j, t}^{W}\right)^{2}+\lambda_{j, t}\left(\epsilon_{j, t, \varepsilon_{j, t}}^{J}\right)^{2}\right]\right\} d t \\
& +\sum_{j=1}^{2}\left\{\int_{0}^{T} 2 \epsilon_{t} \epsilon_{j, t}^{W} d W_{j, t}+\int_{0}^{T} 2 \epsilon_{t^{-}} \epsilon_{j, t}^{J} d J_{j, t}^{*}+\int_{0}^{T}\left[\left(\epsilon_{j, t, \varepsilon_{j, t}}^{J}\right)^{2}-2 \epsilon_{t^{-}} \epsilon_{j, t, \varepsilon_{j, t}}^{J}\right] d N_{j, t}^{*}\right\} .
\end{aligned}
$$

Because $W_{j, t}, N_{j, t}^{*}$ and $J_{j, t}^{*}$ are martingales, we have

$$
\begin{aligned}
\mathbb{E} \epsilon_{T}^{2} & =\mathbb{E} \int_{0}^{T}\left\{2 \rho \epsilon_{1, t}^{W} \epsilon_{2, t}^{W}+\sum_{j=1}^{2}\left[\left(\epsilon_{j, t}^{W}\right)^{2}+\lambda_{j, t}\left(\epsilon_{j, t, \varepsilon_{j, t}}^{J}\right)^{2}\right]\right\} d t \\
& =\mathbb{E} \int_{0}^{T}\left\{2 \rho \epsilon_{1, t}^{W} \epsilon_{2, t}^{W}+\sum_{j=1}^{2}\left[\left(\epsilon_{j, t}^{W}\right)^{2}+\lambda_{j, t} \int_{R}\left(\epsilon_{j, t, y}^{J}\right)^{2} d F_{j}(y)\right]\right\} d t .
\end{aligned}
$$

Differentiating the mean-variance hedging error with respect to $\Delta_{t}$, we can obtain the first-order conditions for the optimal hedging portfolio:

$$
a_{t} \Delta_{t}=b_{t} .
$$

Consequently,

$$
\hat{\Delta}_{t}=\underset{\Delta_{t}}{\operatorname{argmin}} \mathbb{E} \epsilon_{T}^{2}=a_{t}^{-1} b_{t}
$$

\subsection{The Greeks}

Proposition 4.2 Delta is given by

$$
\left\{\begin{array}{l}
\frac{\partial V}{\partial s_{1}}=e^{-q_{1}(T-t)}-\frac{1}{\pi s_{1}} \int_{0}^{\infty} \operatorname{Re}\left[\left(\frac{1}{2}+i w\right) K\left(\frac{1}{2}+i w, \frac{1}{2}-i w, t, T\right)\right] d w \\
\frac{\partial V}{\partial s_{2}}=-\frac{1}{\pi s_{2}} \int_{0}^{\infty} \operatorname{Re}\left[\left(\frac{1}{2}-i w\right) K\left(\frac{1}{2}+i w, \frac{1}{2}-i w, t, T\right)\right] d w
\end{array}\right.
$$

Obviously, $V=s_{1} \frac{\partial V}{\partial s_{1}}+s_{2} \frac{\partial V}{\partial s_{2}}$. Although we allow Hawkes jumps for the pricing model, the relationship between the option value and Delta still holds as indicated in Margrabe (1978) under the Black-Scholes model. Since the option's intrinsic value increases with the price of asset one and decreases with the price of asset two, $\frac{\partial V}{\partial s_{1}}$ is positive and $\frac{\partial V}{\partial s_{2}}$ is negative. 
Proposition 4.3 Theta is given by

$$
\frac{\partial V}{\partial t}=q_{1} s_{1} e^{-q_{1}(T-t)}-\frac{1}{\pi} \int_{0}^{\infty} \operatorname{Re}\left[K^{*}\left(\frac{1}{2}+i w, \frac{1}{2}-i w, t, T\right) K\left(\frac{1}{2}+i w, \frac{1}{2}-i w, t, T\right)\right] d w
$$

where

$$
\begin{aligned}
K^{*}\left(u_{1}, u_{2}, t, T\right)= & \frac{1}{2} u_{1} u_{2}\left(\sigma_{1}^{2}+\sigma_{2}^{2}-2 \rho \sigma_{1} \sigma_{2}\right)+\sum_{j=1}^{2}\left\{\eta_{j} B_{j}^{*}\left(u_{1}, u_{2}, t, T\right)\left(\lambda_{j}-\lambda_{j, \infty}\right)\right. \\
& \left.+\left[1+u_{j} \xi_{j}-\Psi_{j}\left(\gamma_{j} B^{*}\left(u_{1}, u_{2}, t, T\right)\right)\right] \lambda_{j}+u_{j} q_{j}\right\}
\end{aligned}
$$

and $B^{*}\left(u_{1}, u_{2}, t, T\right)=B\left(\left(\mathbf{0}_{V}, u_{1}, u_{2}\right)^{\prime}, t, T\right)$.

If $q_{1}=q_{2}=0$, then the option value decreases with time decay and thus $\frac{\partial V}{\partial t}<0$. However, if the underlying assets pay dividends, the sign of Theta is uncertain.

Proposition 4.4 Vega is

$$
\left\{\begin{array}{l}
\frac{\partial V}{\partial \sigma_{1}}=\frac{\left(\sigma_{1}-\rho \sigma_{2}\right)(T-t)}{\pi} \int_{0}^{\infty}\left(w^{2}+\frac{1}{4}\right) \operatorname{Re}\left[K\left(\frac{1}{2}+i w, \frac{1}{2}-i w, t, T\right)\right] d w \\
\frac{\partial V}{\partial \sigma_{2}}=\frac{\left(\sigma_{2}-\rho \sigma_{1}\right)(T-t)}{\pi} \int_{0}^{\infty}\left(w^{2}+\frac{1}{4}\right) \operatorname{Re}\left[K\left(\frac{1}{2}+i w, \frac{1}{2}-i w, t, T\right)\right] d w
\end{array}\right.
$$

Obviously, the structures of $\frac{\partial V}{\partial \sigma_{1}}$ and $\frac{\partial V}{\partial \sigma_{2}}$ are symmetrical. If $\rho \leq 0$, then the changes in the underlying assets are unrelated or in opposite directions, and higher volatility is more likely to cause higher payoffs and lead to a higher option price; hence, we have $\frac{\partial V}{\partial \sigma_{1}}>0$ and $\frac{\partial V}{\partial \sigma_{2}}>0$. It also implies that the integral part of Vega is positive. However, if $\rho>0$, then the diffusion parts of the underlying assets tend to change in the same direction, and higher volatility do not necessarily cause a larger gap between the prices of two underlying assets. Therefore, the sign of Vega is uncertain.

\section{Proposition 4.5 Gamma is}

$$
\frac{\partial^{2} V}{\partial s_{j}^{2}}=\frac{1}{\pi s_{j}^{2}} \int_{0}^{\infty}\left(w^{2}+\frac{1}{4}\right) \operatorname{Re}\left[K\left(\frac{1}{2}+i w, \frac{1}{2}-i w, t, T\right)\right] d w, \quad j=1,2
$$

Symmetry also arises between $\frac{\partial^{2} V}{\partial s_{1}^{2}}$ and $\frac{\partial^{2} V}{\partial s_{2}^{2}}$, and it follows from Vega that the integral part is positive; thus, $\frac{\partial^{2} V}{\partial s_{1}^{2}}>0$ and $\frac{\partial^{2} V}{\partial s_{2}^{2}}>0$. That is, the option value is a convex function of the price of each underlying asset. Moreover, Rho is $\frac{\partial V}{\partial r}=0$; that is, the value of the exchange option is irrelevant to the risk-free interest rate.

In addition to the usual Greeks, we also obtain the partial derivatives of $V$ with respect to $q_{1}$, 
$q_{2}, \rho$, and $\lambda_{j}$, as follows:

$$
\left\{\begin{array}{l}
\frac{\partial V}{\partial q_{1}}=(T-t)\left\{\frac{1}{\pi} \int_{0}^{\infty} \operatorname{Re}\left[\left(\frac{1}{2}+i w\right) K\left(\frac{1}{2}+i w, \frac{1}{2}-i w, t, T\right)\right] d w-s_{1} e^{-q_{1}(T-t)}\right\} \\
\frac{\partial V}{\partial q_{2}}=\frac{(T-t)}{\pi} \int_{0}^{\infty} \operatorname{Re}\left[\left(\frac{1}{2}-i w\right) K\left(\frac{1}{2}+i w, \frac{1}{2}-i w, t, T\right)\right] d w \\
\frac{\partial V}{\partial \rho}=-\frac{\sigma_{1} \sigma_{2}(T-t)}{\pi} \int_{0}^{\infty}\left(w^{2}+\frac{1}{4}\right) \operatorname{Re}\left[K\left(\frac{1}{2}+i w, \frac{1}{2}-i w, t, T\right)\right] d w \\
\frac{\partial V}{\partial \lambda_{j}}=-\frac{1}{\pi} \int_{0}^{\infty} \operatorname{Re}\left[B_{j}^{*}\left(\frac{1}{2}+i w, \frac{1}{2}-i w, t, T\right) K\left(\frac{1}{2}+i w, \frac{1}{2}-i w, t, T\right)\right] d w, j=1,2 .
\end{array}\right.
$$

Therefore,

$$
\frac{\partial V}{\partial q_{1}}=-(T-t) s_{1} \frac{\partial V}{\partial s_{1}}<0, \quad \frac{\partial V}{\partial q_{2}}=-(T-t) s_{2} \frac{\partial V}{\partial s_{2}}>0, \quad \frac{\partial V}{\partial \rho}=-(T-t) \sigma_{1} \sigma_{2} s_{1}^{2} \frac{\partial^{2} V}{\partial s_{1}^{2}}<0 .
$$

Theoretically, dividend payments decrease the asset price, and the lower the price of asset one (asset two) is, the lower (higher) the value of the exchange option is. Moreover, as the correlation varies from -1 to 1 , the prices of the two underlying assets change from being in opposite directions to being in the same direction, which decreases the intrinsic value of the exchange option gradually. Since a larger jump intensity can make the underlying asset jump more frequently and is more likely to cause a higher (lower) price for asset one (asset two), then the option will likely have a higher payoff, which lead to a higher option price. Hence, we have $\frac{\partial V}{\partial \lambda_{1}}>0$ and $\frac{\partial V}{\partial \lambda_{2}}>0$. Finally, because the partial derivatives of the exchange option value with respective to $\lambda_{j, \infty}, \theta_{j, 1}, \theta_{j, 2}$, and $\eta_{j}$ are not analytical, we will investigate them using a numerical analysis in the following subsection.

\subsection{Numerical Examples}

In the previous discussion, we investigated analytically the sensitivities of the exchange option price with respect to time, the underlying asset prices, volatilities, the correlation coefficient, the dividend rates, the interest rate, the jump intensities, and so on. In this subsection, we use numerical examples to show the relationship between the exchange option price $V_{t}$ and the mean jump sizes, the jump volatilities, and the parameters of the Hawkes intensity process such as $\lambda_{j, \infty}, \theta_{j, 1}, \theta_{j, 2}$, and $\eta_{j}$, and $j=1,2$.

In the numerical examples, we choose jump intensities in the form of (3) and assume that the jump sizes follow normal distributions $\varepsilon_{j, t} \sim N\left(\mu_{j}^{J},\left(\sigma_{j}^{J}\right)^{2}\right), j=1,2$. According to the empirical results of Aït-Sahalia et al. (2015) and Kokholm (2016), the parameter values for the base case are as follows: $t=0, T=1, S_{1,0}=S_{2,0}=1, q_{1}=q_{2}=0, \sigma_{1}=\sigma_{2}=0.15, \rho=0, \mu_{1}^{J}=\mu_{2}^{J}=0$, $\sigma_{1}^{J}=\sigma_{2}^{J}=0.05, \lambda_{1,0}=\lambda_{2,0}=1, \lambda_{1, \infty}=\lambda_{2, \infty}=0.5, \theta_{1,1}=\theta_{2,2}=12.5, \theta_{1,2}=\theta_{2,1}=2.5$, $\eta_{1}=\eta_{2}=20$. In the following numerical analysis, we change the value of only one parameter at a time and keep the values of the remaining parameters unchanged from the base case.

As Figure 1 shows, the sensitivities of the exchange option price to $\mu_{1}^{J}$ and $\mu_{2}^{J}$ are the same, and the value of the exchange option can achieve the minimum when the mean jump size is near zero. In other words, the exchange option price increases with the absolute mean jump sizes, but the effects of positive and negative mean jump sizes with the same absolute value of the exchange option price are not the same. From Figure 2, the sensitivities of the exchange option price to $\sigma_{1}^{J}$ and $\sigma_{2}^{J}$ are also the same, and the exchange option price increases with the jump volatilities.

We can explain the symmetry in Figures 1-2 and the effects of mean jump sizes and jump volatilities on exchange option prices as follows. Assume that there are two exchange options, A and $\mathrm{B}$, and denote by $V_{t}^{A}$ the value of exchange option $A$ with a payoff of $\left(S_{1, T}-S_{2, T}\right)^{+}$and $V_{t}^{B}$ the 

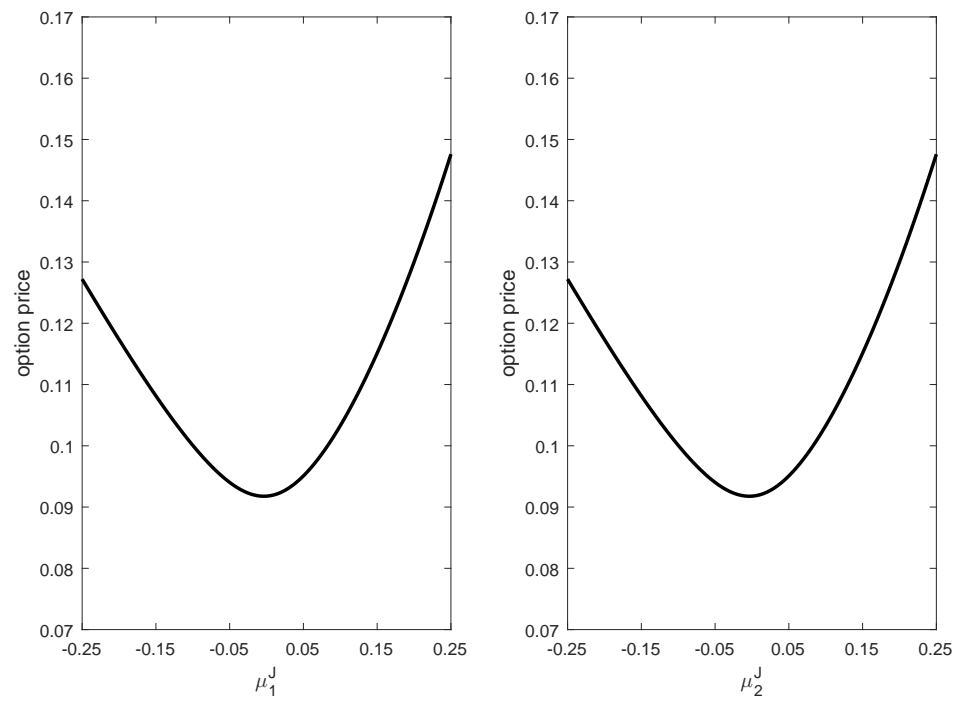

Figure 1. The exchange option price against the mean jump sizes in the Hawkes jump diffusion model.
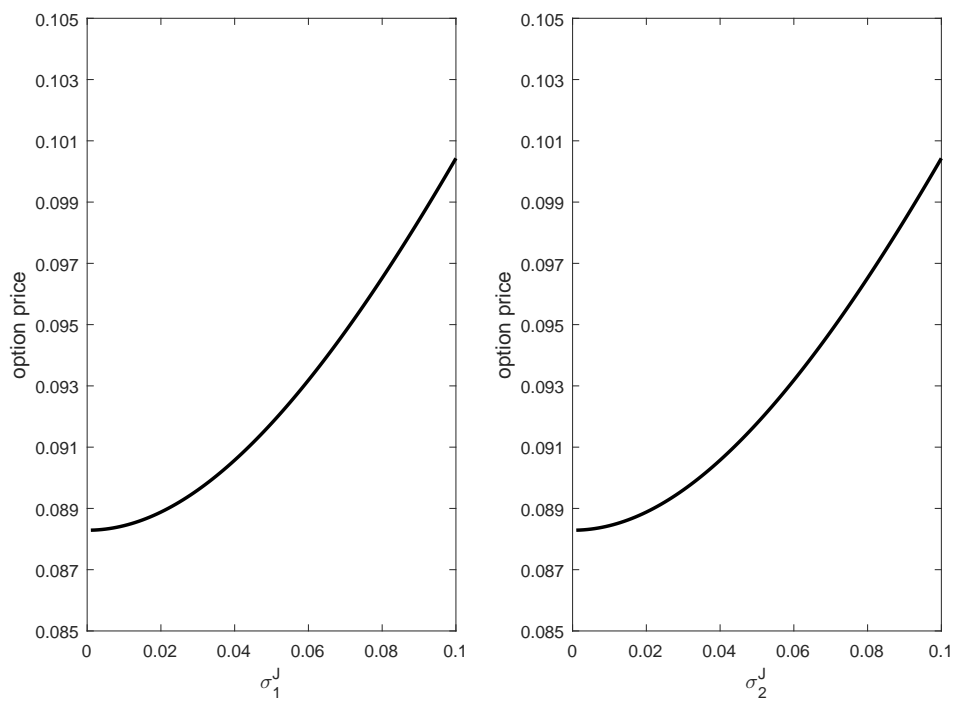

Figure 2. The exchange option price against jump volatilities in the Hawkes jump diffusion model.

value of exchange option $B$ with a payoff of $\left(S_{2, T}-S_{1, T}\right)^{+}$. Since $S_{1, t}$ and $S_{2, t}$ have symmetrical structures, and the payoffs of exchange options $A$ and $B$ are also symmetric, it is obvious that $\frac{\partial V_{t}^{A}}{\partial \mu_{1}^{J}}=\frac{\partial V_{t}^{B}}{\partial \mu_{2}^{J}}$ at $\mu_{1}^{J}=\mu_{2}^{J}$ and $\frac{\partial V_{t}^{A}}{\partial \sigma_{1}^{J}}=\frac{\partial V_{t}^{B}}{\partial \sigma_{2}^{J}}$ at $\sigma_{1}^{J}=\sigma_{2}^{J}$, with the other parameter values set as in the base case. Next, according to European exchange option call-put parity,

$$
V_{t}^{A}+S_{2, t} e^{-q_{2}(T-t)}=V_{t}^{B}+S_{1, t} e^{-q_{1}(T-t)},
$$

we have $\frac{\partial V_{t}^{A}}{\partial \mu_{2}^{J}}=\frac{\partial V_{t}^{B}}{\partial \mu_{2}^{J}}$ and $\frac{\partial V_{t}^{A}}{\partial \sigma_{2}^{J}}=\frac{\partial V_{t}^{B}}{\partial \sigma_{2}^{J}}$. From the above and based on the base case, we have $\frac{\partial V_{t}^{A}}{\partial \mu_{1}^{J}}=\frac{\partial V_{t}^{B}}{\partial \mu_{2}^{J}}=\frac{\partial V_{t}^{A}}{\partial \mu_{2}^{J}}$ at $\mu_{1}^{J}=\mu_{2}^{J}$ and $\frac{\partial V_{t}^{A}}{\partial \sigma_{1}^{J}}=\frac{\partial V_{t}^{B}}{\partial \sigma_{2}^{J}}=\frac{\partial V_{t}^{A}}{\partial \sigma_{2}^{J}}$ at $\sigma_{1}^{J}=\sigma_{2}^{J}$, which means that $\mu_{1}^{J}$ and $\mu_{2}^{J}$ have the same effect on the exchange option price, and so are the effects of $\sigma_{1}^{J}$ and $\sigma_{2}^{J}$. In addition, since the large absolute value of the mean jump size and the large jump volatility can contribute to the fluctuation in the value of the asset, they are all able to cause a high exchange option price. The 

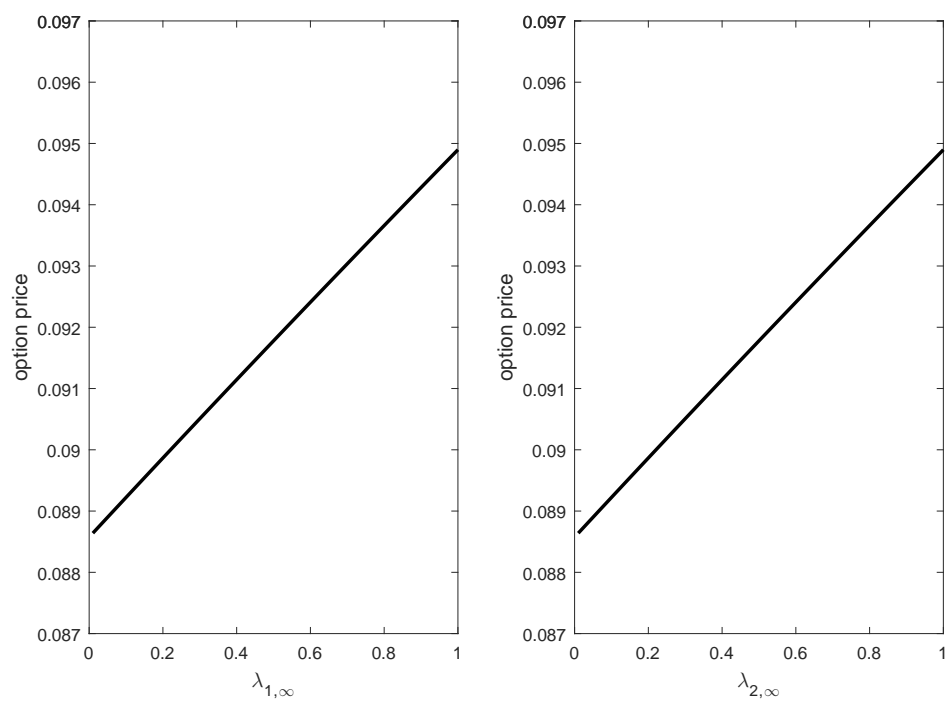

Figure 3. The exchange option price against the intrinsic intensities in the Hawkes jump diffusion model.
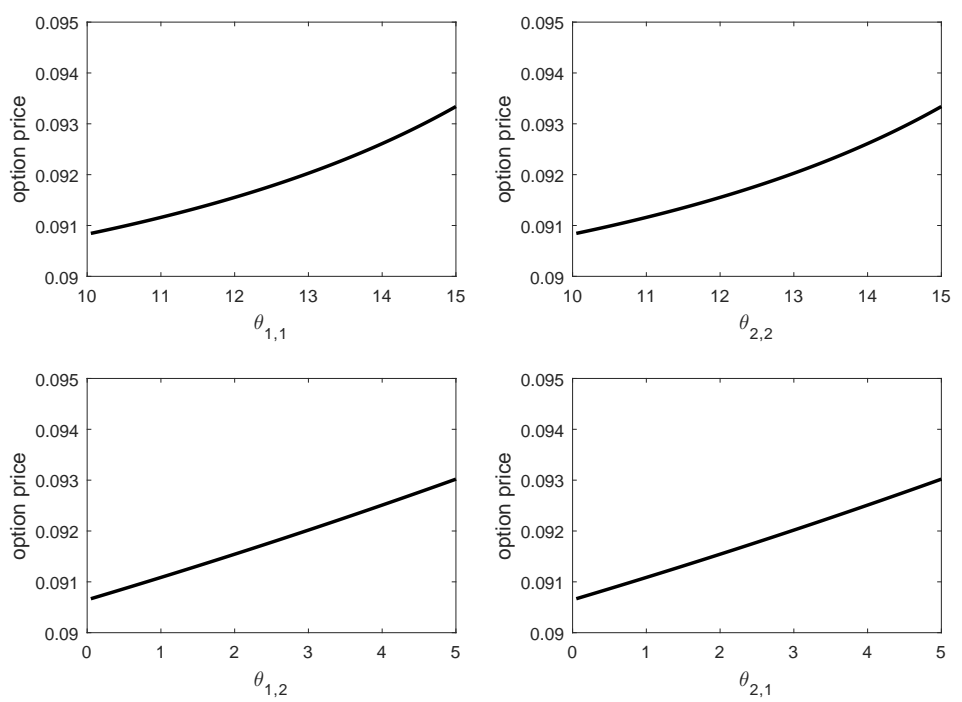

Figure 4. The exchange option price against the jump sizes of the intensities in the Hawkes jump diffusion model.

larger the absolute value of the mean jump sizes or jump volatilities are, the higher the exchange option price is.

Figures 3-5 show the effects of the parameters of the intensity process on the exchange option price. The figures show that the effects of $\lambda_{1, \infty}, \theta_{1,1}, \theta_{1,2}$, and $\eta_{1}$ are the same as that of $\lambda_{2, \infty}, \theta_{2,2}$, $\theta_{2,1}$, and $\eta_{2}$ on the exchange option price, respectively. From Figures 3-4, the exchange option price is increasing in the intrinsic intensities and jump sizes of intensities, respectively. From Figure 5, the exchange option price is decreasing in exponential decay rates. In addition, from Figure 4, the self-excitation coefficients $\left(\theta_{1,1}\right.$ and $\left.\theta_{2,2}\right)$ and the cross-excitation coefficients $\left(\theta_{1,2}\right.$ and $\left.\theta_{2,1}\right)$ make no significant difference in the exchange option price.

We can explain the results in Figures 3-5 as follows. First, larger intrinsic intensities cause more jumps in the asset prices, and then the larger jump sizes of the intensities can increase the intensities when the asset price jumps, which also leads to more jumps in the asset price. Finally, the smaller the exponential decay rates are, the more slowly the increments of the intensities decay and the 

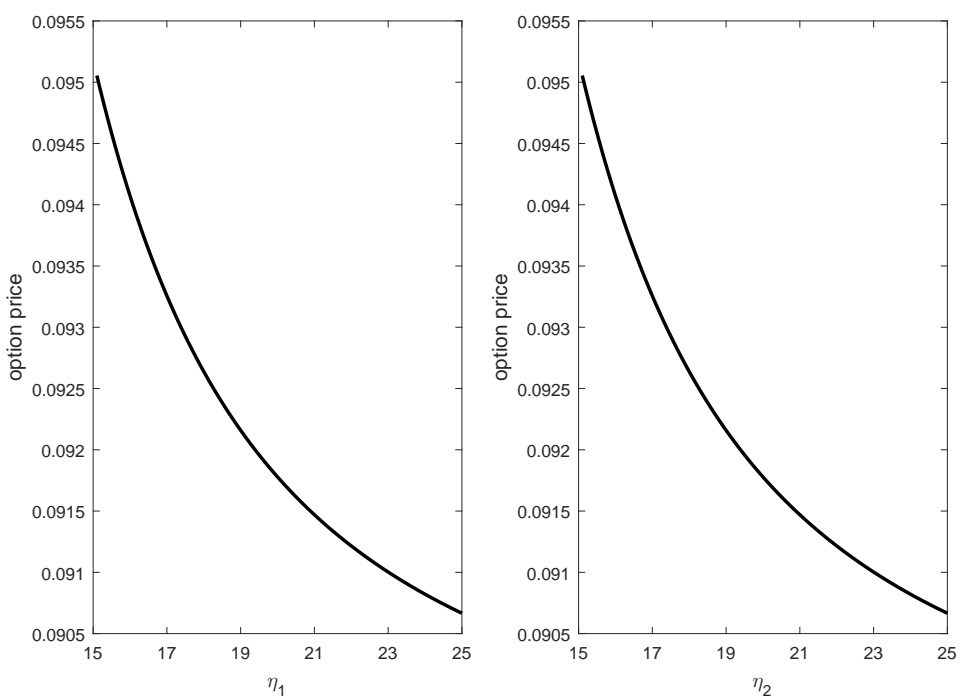

Figure 5. The exchange option price against the exponential decay rates in the Hawkes jump diffusion model.

longer the intensities remain at a high level, which accordingly leads to more jumps in the asset price. In summary, the exchange option price will increase as the asset price jumps more frequently, which can be caused by larger intrinsic intensities, larger jump sizes in the intensities, and smaller exponential decay rates, respectively.

\subsection{Managerial Insights for Arrangements with Exchange Options Characteristics}

In addition to the direct application of evaluating exchange options as an OTC derivative under clustered jumps dynamics, the proposed framework can also apply to exchange-traded derivatives on a single asset with self-excitement clustered jumps. These are special cases since an exchange option degenerates into a vanilla call or put when either asset has constant value over time.

Moreover, the derived valuation formula, Greeks, and optimal hedging strategies offer important managerial insights for many common financial arrangements that assign one party the right to exchange one asset for another in a wide variety of contexts. For instance, in an exchange offer, the target firm's shareholders may exchange their shares for those of the bidding firm as a part or all of the consideration. Hence, as the party that has the option to exchange, the target firm owns an embedded exchange option in the offer. Thus, the value of this exchange option is naturally of interest for the target firm's shareholders. To keep the analysis tractable, assume that this offer conveys no information about the prospects of either firm. Then the offer itself may increase the price of shares in the target firm since its shareholders now have an option to exchange their shares for something else that is worth at least zero, at the cost of the bidding firm's shareholders if the bidding firm does not charge any premium for this tender exchange offer (Margrabe 1978; Amihud et al. 1990).

The rights of the bidding firms to walk away after a deal is announced but before the deal closes is certainly a very interesting addition to the literature. Gilson and Schwartz (2005) study merger contracts that contain material adverse change (MAC) and material adverse effect (MAE) clauses that allow the buyer to costlessly cancel the deal if such a change or effect occurs. They examine the litigation functions that the MAC and MAE term serve in terms of the threat of moral hazard in the structure of an M\&A agreement. Denis and Macias (2013) show that MAEs are the underlying cause of $69 \%$ of acquisition terminations and $80 \%$ of renegotiations, and acquisitions with fewer MAE exclusions are associated with higher offer premiums, which clearly provides evidence that MAE clauses contain substantial value as an option. Bhagwat et al. (2016) study the 
general risk of either party walking away. They find empirically that increases in market volatility decrease subsequent deal activity, and merging parties attempt to shorten the interim window as risk increases. These empirical observations are consistent with the implications of viewing the right to walk away as an exchange option, the value of which would increase when firm values can change substantially, and decrease when the interim window between the time deal terms are set and the actual deal closing are shortened.

Obviously, when there are possible clustered jumps for either the bidding or target firm, or across the two firms, the intensity and size of jumps and other parameters would affect the value increase of the target firm, as we discussed. Moreover, when the bidding firm is a public company, it may attempt to issue more stocks as a way to finance the acquisition, in which case, the value of such exchange offer would affect more public investors. A further complication that can arise in valuing these opportunities occurs when the exchange offer also includes a bringdown condition, such that the bidding firm can walk away when the target firm's stock price drops significantly relative to that of the bidding firm between signing and closing. We can consider such walk away covenant as an exchange option owned by the bidding firm, and will reduce the value of the exchange option owned by the target firm. Managers of either firm or hedge funds that want to speculate on the M\&A transaction, may adopt some hedging strategies based on the proposed framework to help mitigate the risk from the change in the exchange option value due to the clustered jump dynamics.

As another example, the proposed framework may also shed some light on the value of portfolio managers. Passive investment that tracks some major market indices such as the Standard and Poor's 500 Index using an exchange-traded fund (ETF) or index fund is both cost effective and can be easily self-managed by the investors themselves. Portfolio or fund managers offer services to provide an opportunity to outperform the broad market indices at a nontrivial cost, so it is important to evaluate fund managers and mitigate risk. Investors may be concerned about the probability that a managed portfolio will underperform against the market benchmark. Since the managed portfolio can be considered as exchanging at least partial components of the passive investments in the benchmark with actively managed investments, investors may consider an equity swaption as having the right to exchange the deviations back to the original components in the benchmark, such that the investors can always get the higher return from the managed portfolio and the market benchmark. The value of the portfolio manager can be considered as the difference in returns from the managed portfolio and premium of the equity swaption. Due to the potential spread of market disturbances, it is important to incorporate clustered jumps in a portfolio manager evaluation.

With a similar concept, Cremers and Petajisto (2009) and Petajisto (2013) introduce active shares as a simple measure of the a fund manager's activeness by looking at the absolute deviations of the manager's portfolio holdings against their benchmark portfolio holdings and relate active share to fund characteristics such as size, expense, and turnover in the cross-section. Despite its simplicity, Frazzini et al. (2016) more recently suggest that the active share is not a valuable measure of managers' skill, as it cannot justify the value of such deviations. In comparison, the proposed framework could be used to directly evaluate the option value of the manager's portfolio holdings against their benchmark portfolio holdings by considering the deviations as exchange options.

While the complete development of these ideas is beyond the scope of this paper, we are optimistic that they will be subjects of future research that extends this work.

\section{Implied Beta Under Clustered Jumps}

In this section, we discuss the implied beta under the proposed Hawkes jump-diffusion model and compare the implied betas under the Hawkes jump-diffusion model, Poisson jump-diffusion model, and Black-Scholes model (geometric Brownian model). 


\subsection{Implied Beta}

Systematic risk plays a critical role in finance practice. Beta is the well-known measure of the systematic risk of an asset. Beta is the variability of the asset's return relative to the return of the market portfolio. In practice, market indexes are usually used to represent the market portfolio. The traditional method to estimate beta is typically based on a regression model using the historical returns of the asset and the market portfolio. For instance, according to the CAPM,

$$
R_{X, t}=\alpha_{t}+\beta_{t} R_{M, t}+e_{t},
$$

where $R_{X, t}$ and $R_{M, t}$ are rates of return from the asset and market, respectively, and $e_{t}$ is a random error with zero-mean and is independent of $R_{M, t}$. Beta $\beta_{t}$ is given by

$$
\beta_{t}=\frac{\operatorname{Cov}\left(R_{X, t}, R_{M, t}\right)}{\operatorname{Var}\left(R_{M, t}\right)} .
$$

However, it has long been recognized that significant bias can arise when using historical data to estimate the expected model since it leads to sensitivity to minor changes in the sample period and the choices of single or multi-factor models (Sharpe 1964; Fama and MacBeth 1973; Fama and French 1993; McNulty et al. 2002). In essence, it is hard to assess the bias associated with the estimation of an ex ante model using the ex post historical data.

More recently, several attempts have been made to reduce the estimation error that arises from the use of historical data by exploiting information about systematic risk contained in option prices. For instance, Chang et al. (2011) use both option-implied skewness and volatility to estimate market beta. Buss and Vilkov (2012) use stock returns in the market index constituents and a factor model to construct a complete correlation matrix. However, these estimation methods rely on simplified assumptions, such as a single factor model, zero skewness of the market return residual, or unrealistic assumptions such as that the implied correlations are always higher than the realized correlations. Despite these weak assumptions, these methods show the significant explanatory power of information from the option market on estimating beta (Harris et al. 2019).

Under the Black-Scholes model, Siegel (1995) demonstrates a method to derive the implied beta from an exchange option for which the underlying assets are the market index and the asset, and two vanilla options on the market index and the asset, respectively. In the following, we show how to derive the implied beta from the exchange options under the proposed Hawkes jump-diffusion model.

THEOREM 5.1 The implied beta under the Hawkes jump-diffusion model is

$$
\beta_{t}=\frac{\rho \sigma_{X} \sigma_{M}}{\sigma_{M}^{2}+\zeta_{M} \frac{\lambda_{M, \infty} \eta_{M}\left(\eta_{X}-\theta_{X, X}\right)+\lambda_{X, \infty} \eta_{X} \theta_{M, X}}{\left(\eta_{M}-\theta_{M, M}\right)\left(\eta_{X}-\theta_{X, X}\right)-\theta_{M, X} \theta_{X, M}}} .
$$

Proof. Let $S_{M, t}$ be the market index price and $S_{X, t}$ be the asset price. For consistency with our proposed framework and notations, we simply replace the subscripts 1 and 2 with $X$ and $M$, respectively; that is, the individual asset is asset 1 and the market index is asset 2 in the exchange option. The rates of return are

$$
R_{X, t} d t=\frac{d S_{X, t}}{S_{X, t}}, \quad R_{M, t} d t=\frac{d S_{M, t}}{S_{M, t}} .
$$

Thus, we have

$$
\beta_{t}=\frac{\operatorname{Cov}\left(d S_{X, t} / S_{X, t}, d S_{M, t} / S_{M, t}\right)}{\operatorname{Var}\left(d S_{M, t} / S_{M, t}\right)} .
$$


Note that, since the variance and covariance are not subject to adaptation with the change in measure, we calculate them under the risk-neutral measure. Then, under the risk-neutral measure, the asset price and the market index follow the dynamics:

$$
\left\{\begin{array}{l}
\frac{d S_{X, t}}{S_{X, t}}=\left(r-q_{X}\right) d t+\sigma_{X} d W_{X, t}+d J_{X, t}^{*} \\
\frac{d S_{M, t}}{S_{M, t}}=\left(r-q_{M}\right) d t+\sigma_{M} d W_{M, t}+d J_{M, t}^{*}
\end{array}\right.
$$

Obviously,

$$
\begin{gathered}
\operatorname{Var}\left(d S_{M, t} / S_{M, t}\right)=\sigma_{M}^{2} d t+\operatorname{Var}\left(d J_{M, t}^{*}\right), \\
\operatorname{Cov}\left(d S_{X, t} / S_{X, t}, d S_{M, t} / S_{M, t}\right)=\rho \sigma_{X} \sigma_{M} d t+\operatorname{Cov}\left(d J_{X, t}^{*}, d J_{M, t}^{*}\right) .
\end{gathered}
$$

Since $\operatorname{Var}\left(d J_{M, t}^{*}\right)=\zeta_{M} \mathbb{E} \lambda_{M, t} d t+o(d t)$ and $\operatorname{Cov}\left(d J_{X, t}^{*}, d J_{M, t}^{*}\right)=o(d t)$, we have

$$
\beta_{t}=\frac{\rho \sigma_{X} \sigma_{M}}{\sigma_{M}^{2}+\zeta_{M} \mathbb{E} \lambda_{M, t}}
$$

According to Aït-Sahalia et al. (2015),

$$
\mathbb{E} \lambda_{M, t}=\frac{\lambda_{M, \infty} \eta_{M}\left(\eta_{X}-\theta_{X, X}\right)+\lambda_{X, \infty} \eta_{X} \theta_{M, X}}{\left(\eta_{M}-\theta_{M, M}\right)\left(\eta_{X}-\theta_{X, X}\right)-\theta_{M, X} \theta_{X, M}}
$$

thus, the implied beta under the Hawkes jump-diffusion model is

$$
\beta_{t}=\frac{\rho \sigma_{X} \sigma_{M}}{\sigma_{M}^{2}+\zeta_{M} \frac{\lambda_{M, \infty} \eta_{M}\left(\eta_{X}-\theta_{X, X}\right)+\lambda_{X, \infty} \eta_{X} \theta_{M, X}}{\left(\eta_{M}-\theta_{M, M}\right)\left(\eta_{X}-\theta_{X, X}\right)-\theta_{M, X} \theta_{X, M}}} .
$$

\subsection{Simulation Study of Implied Betas under Different Models}

To calculate the implied beta under the proposed model, we first jointly calibrate all of the parameters except $\rho$ to the prices of the market-traded plain vanilla options on the market index and on the asset. Second, based on the pricing formula in (15), we deduce $\rho$ from the exchange option price whenever it is tradable. Specifically, if we replace $S_{2, t}$ with strike price $K, q_{2}$ with interest rate $r, K_{3}(1 / 2+i w, 1 / 2-i w, t, T)$ with $K_{3}(1 / 2+i w, 0, t, T)$, and set $\rho, \sigma_{2}, \lambda_{2, t}$, and the parameters of the jump size distribution in the asset two equal to zero, then we will transform (15) into the pricing formula for European call options on asset one with strike $K$ at time $t$. Using this procedure, we can derive the implied betas by the observed exchange option prices. Since the Hawkes jump diffusion model includes the Black-Scholes model as a special case and allows jump contagion and jump clustering, which are empirically detected in reality, the proposed model could derive a more accurate forward-looking beta.

We first consider a hypothetical example by generating a series of prices on the market index and on the asset for European options with different strike prices to represent the real market-traded option prices. To generate European call option prices, we assume that the distributions of the jump sizes of the market index and the asset are the same as those in section 5.1, and set the parameters for the base case as follows: $t=0, T=0.25, S_{M, 0}=S_{X, 0}=1, r=0.05, q_{M}=q_{X}=0$, $\sigma_{M}=0.2, \sigma_{X}=0.25, \mu_{M}^{J}=\mu_{X}^{J}=0, \sigma_{M}^{J}=\sigma_{X}^{J}=0.05, \lambda_{M, 0}=\lambda_{X, 0}=\lambda_{M, \infty}=\lambda_{X, \infty}=1$, $\theta_{M, M}=\theta_{X, X}=12.5, \theta_{M, X}=\theta_{X, M}=2.5$, and $\eta_{M}=\eta_{X}=20$. We choose ten different strike prices 

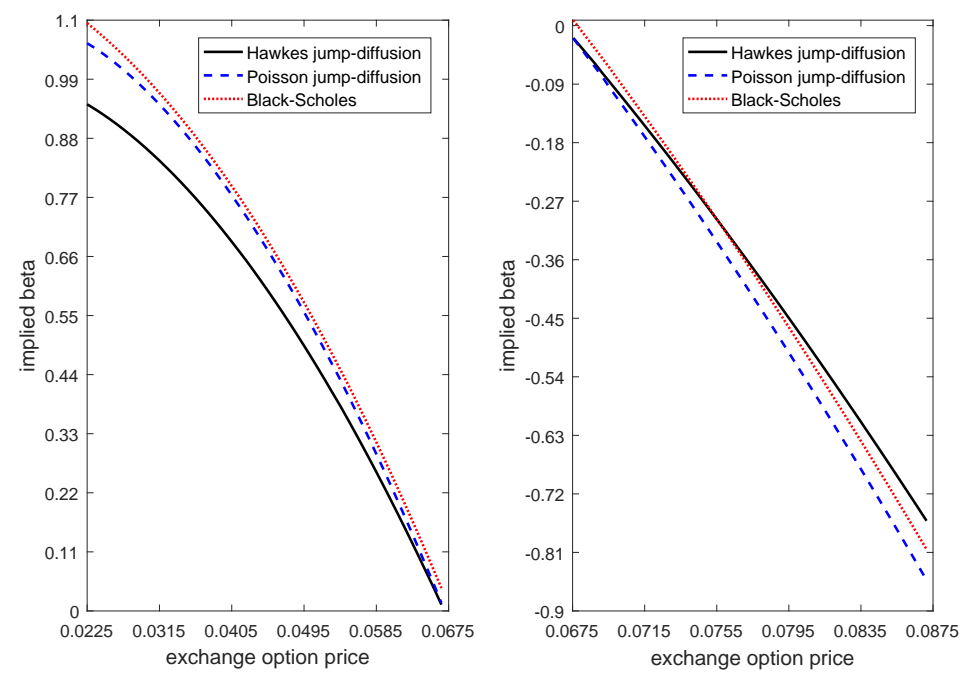

Figure 6. Implied beta against the exchange option price under the Black-Scholes model, Poisson jump-diffusion, and Hawkes jump-diffusion models.
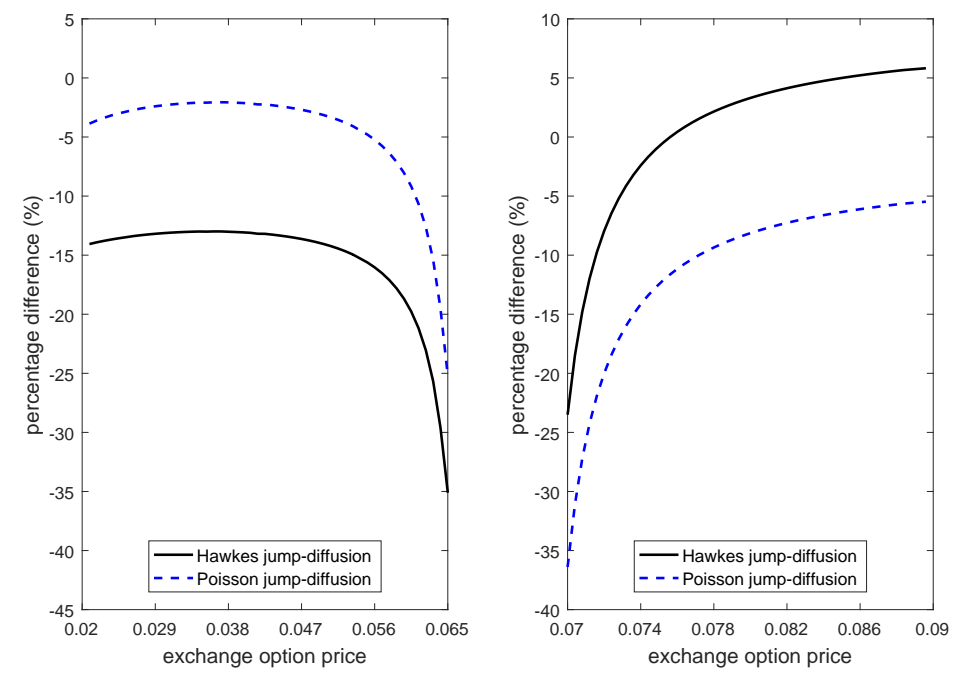

Figure 7. The percentage difference between different model-implied betas with the Black-Scholes model-implied beta as the benchmark. The left and right plots represent the positive and negative benchmarks, respectively.

of $0.8,0.85,0.9,0.95,1,1.05,1.1,1.15,1.2$, and 1.25, and calculate the corresponding European call option prices on the market index and on the asset, respectively. Table 1 reports the results.

We then apply these option prices to jointly calibrate the parameters except $\rho$ under the Hawkes jump-diffusion model, Poisson jump-diffusion model, and Black-Scholes model, respectively, with detailed calibration procedure to be discussed in subsection 6.2. The parameters $t, T, S_{M, t}, S_{X, t}$, $r, q_{M}$, and $q_{X}$ remain unchanged from the base case. We summarize the calibrated parameters in Table 2. Finally, we deduce $\rho$ from the exchange option price under the three models above respectively, and then obtain the implied betas $\beta^{H}, \beta^{P}$, and $\beta^{B}$ with (28) and (29), where the superscripts refer to the initial letters of these models.

Figure 6 shows that the implied betas are decreasing in the exchange option price under these three models, and that $\beta^{P}<\beta^{B}$ and the gap between $\beta^{P}$ and $\beta^{B}$ decreases first and increases later with the increasing exchange option price. In addition, there exists two exchange option prices at which we have $\beta^{H}=\beta^{P}$ and $\beta^{H}=\beta^{B}$. We denote the two exchange option prices as $V_{1}$ and $V_{2}$, and 
Table 1. European call option prices with different strike prices

\begin{tabular}{cccccc}
\hline Strike price & 0.8 & 0.85 & 0.9 & 0.95 & 1 \\
Index option price & 0.2105 & 0.1627 & 0.1180 & 0.0791 & 0.0485 \\
Equity option price & 0.2116 & 0.1656 & 0.1234 & 0.0871 & 0.0580 \\
\hline Strike price & 1.05 & 1.1 & 1.15 & 1.2 & 1.25 \\
Index option price & 0.0271 & 0.0139 & 0.0066 & 0.0029 & 0.0013 \\
Equity option price & 0.0364 & 0.0216 & 0.0121 & 0.0065 & 0.0034 \\
\hline
\end{tabular}

Table 2. Calibrated parameters under three different models

\begin{tabular}{cccccccc}
\hline Parameters & $\sigma_{M}$ & $\sigma_{X}$ & $\lambda_{M, \infty} / \lambda_{M}$ & $\lambda_{X, \infty} / \lambda_{X}$ & $\theta_{M, M}$ & $\theta_{M, X}$ & $\theta_{X, M}$ \\
Hawkes J-D model & 0.2003 & 0.2481 & 0.7946 & 1.3452 & 11.5337 & 3.5402 & 3.8935 \\
Poisson J-D model & 0.2026 & 0.2420 & 0.4410 & 2.5604 & - & - & - \\
B-S model & 0.2169 & 0.2620 & - & - & - & - & - \\
\hline Parameters & $\theta_{X, X}$ & $\eta_{M}$ & $\eta_{X}$ & $\mu_{M}^{J}$ & $\mu_{X}^{J}$ & $\sigma_{M}^{J}$ & $\sigma_{X}^{J}$ \\
Hawkes J-D model & 8.7502 & 22.7006 & 13.6142 & -0.0002 & -0.0002 & 0.0545 & 0.0477 \\
Poisson J-D model & - & - & - & -0.0045 & 0.0015 & 0.1033 & 0.0607 \\
B-S model & - & - & - & - & - & - & - \\
\hline
\end{tabular}

$V_{1}<V_{2}$. When the exchange option price is lower than $V_{1}$, we have $\beta^{H}<\beta^{P}<\beta^{B}$, and both the gap between $\beta^{H}$ and $\beta^{P}$ and that between $\beta^{H}$ and $\beta^{B}$ are decreasing in the exchange option price. When the exchange option price is higher than $V_{1}$ but lower than $V_{2}$, we have $\beta^{P}<\beta^{H}<\beta^{B}$, and with an increasing exchange option price, the gap between $\beta^{H}$ and $\beta^{P}$ increase and that between $\beta^{H}$ and $\beta^{B}$ decrease. When the exchange option price is higher than $V_{2}$, we have $\beta^{P}<\beta^{B}<\beta^{H}$, and both the gap between $\beta^{H}$ and $\beta^{P}$ and that between $\beta^{H}$ and $\beta^{B}$ are increasing in the exchange option price.

Moreover, to present the relative difference in the implied betas between the jump-diffusion model and the Black-Scholes model, we take $\beta^{B}$ as the benchmark to calculate the percentage difference (PD) between $\beta^{H}$ and $\beta^{B}$, and that between $\beta^{P}$ and $\beta^{B}$, which we define as $P D_{j}=\left(\beta^{j}-\beta^{B}\right) /\left|\beta^{B}\right|$ to ensure that its sign is consistent with $\beta^{j}-\beta^{B}$, where $j \in\{H, P\}$. It is easy to deduce that $P D_{P}<0$ and to infer from Figure 6 that when the exchange option price is lower than $V_{2}$, $P D_{H}<0$, we have $\left|P D_{H}\right|<\left|P D_{P}\right|$ if $\beta^{H}<\beta^{P}$ and vice versa. When the exchange option price is higher than $V_{2}, P D_{H}>0$ and the change in $P D_{H}$ and $\left|P D_{H}\right|$ is the same. Figure 7 shows that both $P D_{H}$ and $P D_{P}$ increase slightly first and then decrease if $\beta^{B}>0$, and increase if $\beta^{B}<0$, with the increasing exchange option price. In addition, when the exchange option price is higher than $V_{2},\left|P D_{H}\right|$ is smaller than $\left|P D_{P}\right|$ at first, and then as the exchange option price increases, the gap between $\left|P D_{H}\right|$ and $\left|P D_{P}\right|$ decreases gradually to zero, and afterward $\left|P D_{H}\right|$ is larger than $\left|P D_{P}\right|$.

\subsection{Economic Implications of the Implied Betas under Clustered Jumps}

The benefits of the implied beta are analogous to those of the implied volatility that directly indicates the current market sentiment on systematic risk. The proposed framework captures higher moments and tail dependency due to the clustered jumps, and can hence provide extra information. As illustrated in Figures 6 and 7, ignoring clustered jumps may significantly misestimate the implied beta, which can be quantified by comparing the results. Because systematic risk changes through time, especially in volatile and contagious market environments, an option-implied beta would provide timely and improved estimates of forward-looking systematic risk and reflect the markets' expectation of beta, incorporating the clustered jumps over the life of the exchange option.

The better predictive quality of the implied betas under clustered jumps within our proposed 
framework could lead to better cost-of-capital estimates and may have numerous empirical applications, such as detecting abnormal returns and statistical arbitrage in asset pricing and risk management in cases when clustered jumps could be a concern or investment opportunity.

\section{Hybrid Procedure for Implied Beta}

As an OTC market product, exotic options prices are usually only available to institutional investors who can request quotes from market makers to estimate the implied beta with the proposed framework. For retail investors who lack access to the exchange option prices, we also propose an alternative procedure. French et al. (1983) and Chang et al. (2011) explore methods of combining option-implied volatility with historical correlations to improve the measurement of betas. In addition, Buss and Vilkov (2012) propose option-implied correlations based on the Black-Scholes framework. Despite the obvious limitations, these studies show that the use of option-implied volatility, and historical or option-implied correlations, exhibit smaller and less systematic prediction errors in their beta estimations.

In the same spirit, we propose a hybrid approach by combining the jointly calibrated optionimplied parameters in the Hawkes processes from the market-traded options and the historical covariances from the stock and market index historical returns when investors do not have access to the exchange options prices. In this section, we estimate the historical betas and the optionimplied betas under the Hawkes jump-diffusion model and the Poisson jump-diffusion model using market data, and examine the performance of the various estimates to predict the future beta.

\subsection{Data}

We obtained the options data from the OptionMetrics database for two types of financial market environments: the financial crisis period from January 2008 to December 2009 and the relatively steady period from January 2016 to December 2017. Following the literature (c.f. Chang et al. 2011; Samuel 2018), we eliminated options that violated the arbitrage bounds, have zero volume, zero open interest, or missing quotes from the sample to ensure an accurate calculation of the parameters. We likewise excluded all observations for which the ask price was below the bid price, the bid price was equal to zero, or the bid-ask spread was below the minimum tick size. As in Buss and Vilkov (2012) and Harris et al. (2019), we use the mid closing bid-ask prices as the option prices and choose the options with the last expiration date within each month when there are one or more options expiration dates. Following Harris et al. (2019), we estimate the historical beta and the option-implied beta on a monthly basis. We use the daily returns on the stock and market index in the historical period to estimate covariance and to calculate the historical beta. We use the daily returns of the stock and market index in the forward-looking period of 7 weeks to calculate the future realized beta as the forecast benchmark. The S\&P 500 index (ticker symbol SPX) serves as a proxy for the U.S. market. In addition, we use the treasury bill yield as a proxy for the risk-free interest rate, which we obtain from the CRSP treasury database.

Since the 2008 financial crisis originated from the financial industry and has a greater clustered impact on the financial industry than on the other industries, we choose eight stocks from the financial industry: JP Morgan Chase (JPM), Bank of America (BAC), Citigroup (C), Wells Fargo (WFC), Goldman Sachs (GS), Morgan Stanley (MS), U.S. Bancorp (USB), and Capital One (COF). These companies are among the largest banking firms with the most actively traded derivatives in the U.S. ${ }^{1}$.

\footnotetext{
${ }^{1}$ TD Bank and PNC Financial Services are among the largest banks, but the option expiration dates for these two options are very limited. Hence, we exclude these two financial companies in our empirical study.
} 


\subsection{Model Calibration, Beta Calculation, and Prediction Evaluation}

We jointly calibrate the parameters of option-implied beta using the vanilla option prices of the stock and market index, except for the covariance by using the historical returns on the stock and market index in the hybrid approach. More specifically, we calibrate the option model by minimizing the sum of squared errors (SSE) for the implied volatilities of each model and the traditional implied volatility across strike prices. The calibration objective function is

$$
S S E=\sum_{\text {options }}\left(I V^{M}-I V^{R}\right)^{2}, \quad M \in\{H, P\} .
$$

where $I V^{R}$ is the traditional implied volatility, and $I V^{H}$ and $I V^{P}$ are the Hawkes model and Poisson model implied volatility, respectively. Since the implied volatility corresponds to the option price via the Black-Scholes-type model, we can derive the implied volatility from option price. For a call option with strike price $K$ and underlying asset price $S_{1, t}$, which satisfies the dynamic in Section 2, the value of the call option under the Hawkes jump-diffusion model is

$$
V_{t}=S_{1, t} e^{-q_{1}(T-t)}-\frac{1}{\pi} \int_{0}^{\infty} \operatorname{Re}\left[\Gamma\left(\frac{1}{2}+i w, \frac{1}{2}-i w, t, T\right)\right] d w
$$

where

$$
\Gamma\left(u_{1}, u_{2}, t, T\right)=\frac{1}{u_{1} u_{2}} \exp \left\{\sum_{j=1}^{2} \Gamma_{j}\left(u_{1}, u_{2}, t, T\right)+\Gamma_{3}\left(u_{1}, t, T\right)\right\}
$$

and

$$
\left\{\begin{array}{l}
\Gamma_{1}\left(u_{1}, u_{2}, t, T\right)=u_{1}\left[\log S_{1, t}-q_{1}(T-t)\right]+u_{2}[\log K-r(T-t)] \\
\Gamma_{2}\left(u_{1}, u_{2}, t, T\right)=-\frac{1}{2} u_{1} u_{2} \sigma_{1}^{2}(T-t) \\
\Gamma_{3}\left(u_{1}, t, T\right)=A^{*}\left(u_{1}, 0, t, T\right)+B_{1}^{*}\left(u_{1}, 0, t, T\right) \lambda_{1, t}
\end{array}\right.
$$

If $\theta_{i, j}=0, i, j \in\{1,2\}$, then the Hawkes model will degenerate to a Poisson model. We assume that the market index and stock price dynamics are the same as those in Section 5.1, and that $q_{1}=q_{2}=0$. To evaluate the calibration performance, we use the mean absolute error (MAE), defined as

$$
\mathrm{MAE}^{M}=\frac{1}{\#\{\text { options }\}} \sum_{\text {options }}\left|I V^{M}-I V^{R}\right|, \quad M \in\{H, P\}
$$

Clearly, the smaller the mean absolute error is, the better the calibration performance is. Following French et al. (1983) and Harris et al. (2019), we assume that the covariance between the market index and stock remain unchanged in the short term so that we can use the historical covariance to replace the option-implied covariance. Let $R_{M, \text { hist }}$ and $R_{X, h i s t}$ be the historical returns, $R_{M, f u t}$ and $R_{X, f u t}$ be the future returns, and $\sigma_{M}, \sigma_{X}$ and $\sigma_{M, X}$ be the option-implied volatilities and covariance. Then, the implied correlation coefficient is

$$
\rho=\frac{\sigma_{M, X}}{\sigma_{M} \sigma_{X}}=\frac{\operatorname{Cov}\left(R_{M, h i s t}, R_{X, h i s t}\right)}{\sigma_{M} \sigma_{X}}=\frac{n \sum R_{M, h i s t} R_{X, h i s t}-\sum R_{M, h i s t} \sum R_{X, h i s t}}{n^{2} \sigma_{M} \sigma_{X}} .
$$

Using the daily returns in the historical period and the option-implied volatilities, we can estimate the correlation coefficient, and finally, calculate the implied betas by Equation (29). Additionally, 
we calculate the historical beta $\beta_{\text {hist }}$ and future realized beta $\beta_{f u t}$ as

$$
\begin{aligned}
& \beta_{\text {hist }}=\frac{\operatorname{Cov}\left(R_{M, \text { hist }}, R_{X, \text { hist }}\right)}{\operatorname{Var}\left(R_{M, h i s t}\right)}=\frac{n \sum R_{M, \text { hist }} R_{X, \text { hist }}-\sum R_{M, \text { hist }} \sum R_{X, \text { hist }}}{n \sum R_{M, \text { hist }}^{2}-\left(\sum R_{M, \text { hist }}\right)^{2}}, \\
& \beta_{f u t}=\frac{\operatorname{Cov}\left(R_{M, f u t}, R_{X, f u t}\right)}{\operatorname{Var}\left(R_{M, f u t}\right)}=\frac{n \sum R_{M, f u t} R_{X, f u t}-\sum R_{M, f u t} \sum R_{X, f u t}}{n \sum R_{M, f u t}^{2}-\left(\sum R_{M, f u t}\right)^{2}} .
\end{aligned}
$$

Let $\beta_{i m p}^{M}$ be the implied beta under model $M, \quad M \in\{H, P\}$. We can also use the MAE to evaluate the prediction ability. We define the MAE of the prediction as

$$
\mathrm{MAE}_{\text {hist }}=\frac{1}{\#\{\text { months }\}} \sum_{\text {months }}\left|\beta_{\text {hist }}-\beta_{\text {fut }}\right|, \quad \mathrm{MAE}_{\text {imp }}^{M}=\frac{1}{\#\{\text { months }\}} \sum_{\text {months }}\left|\beta_{\text {imp }}^{M}-\beta_{\text {fut }}\right| .
$$

The smaller the MAE is, the more accurate the prediction is. Since the option-implied betas contain forward-looking information, we expect that the prediction ability of the option-implied betas should be better than that of the historical beta.

\subsection{Empirical Results}

Using the option data of the S\&P 500 index and the 8 stocks with different strike prices, we jointly calibrate the option-implied betas in each month during the financial crisis and the steady period, and under the Hawkes and the Poisson jump-diffusion models, respectively.

Table 3 reports the prediction performance of the different types of betas during the two sample periods. It is clear that the prediction error of the implied beta under the Hawkes jump-diffusion model is always the smallest, the implied beta under the Poisson model is the next accurate, and the prediction errors of the historical betas are always larger than that of both implied betas, in either sample period. First, this result shows that the implied beta is a better estimator than the historical beta is, and the forward-looking information in option data reveals extra information about the future beta. Second, since the Hawkes jump-diffusion model can capture the characteristic of selfand cross-excitation, it more accurately describes the underlying asset dynamics and the jump interaction between the two assets. Thus, the implied beta under the Hawkes model has a smaller prediction error than that under the Poisson model. Third, in either the financial crisis period or the steady period, if one stock has the largest prediction error for the historical beta, then the prediction error of the implied beta also tend to be the largest, and vice versa. Since the larger prediction error of the historical beta implies the existence of larger changes in the covariance or volatilities, and under our hybrid approach for implied beta, a larger change in covariance will lead to a larger error in estimating implied correlation coefficient, which in turn would lead to a larger prediction error for the implied beta. Therefore, the complete option-implied betas from tradable exchange options will potentially outperform those from the hybrid approach, since the tradable exchange options will provide a better estimation of the correlation coefficients.

We further define the prediction improvement rate (PIR) of a beta estimate relative to another as

$$
\mathrm{PIR}=\frac{\left|\mathrm{MAE}_{2}-\mathrm{MAE}_{1}\right|}{\mathrm{MAE}_{1}}
$$

where $M A E_{i}, i=1,2$ are the mean absolute errors with respect to the prediction of the two betas. Table 3 has shown that option-implied beta has stronger predication power than the historical beta in both periods. Panels A and B in Table 4 further reveal that the prediction performance of the option-implied beta relative to the historical beta is even better during the financial crisis period in comparison to the relatively steady period. This is intuitive since the forward-looking 
Table 3. The predicted MAEs of the historical beta and implied betas under the Hawkes model and Poisson model for different stocks during two distinct time periods

\begin{tabular}{cccccccccc}
\hline Beta & Period & JPM & BAC & C & WFC & GS & MS & USB & COF \\
\hline \multirow{2}{*}{$\beta_{i m p}^{H}$} & $2008-2009$ & 0.4589 & 0.6408 & 0.5863 & 0.5806 & 0.3636 & 0.4335 & 0.6006 & 0.6553 \\
\multirow{2}{*}{$\beta_{i m p}^{P}$} & $2016-2017$ & 0.4285 & 0.6417 & 0.5747 & 0.3890 & 0.5409 & 0.7257 & 0.3058 & 0.4346 \\
\multirow{2}{*}{$\beta_{\text {hist }}$} & $2016-2009$ & 0.5241 & 0.7064 & 0.6334 & 0.6327 & 0.4145 & 0.4710 & 0.6494 & 0.6985 \\
& $2008-2009$ & 0.4840 & 0.6670 & 0.6029 & 0.4064 & 0.5707 & 0.7402 & 0.3189 & 0.4594 \\
& $2016-2017$ & 0.5154 & 0.8771 & 0.6769 & 0.8277 & 0.4412 & 0.5238 & 0.6809 & 0.7579 \\
\hline
\end{tabular}

Table 4. The PIRs (\%) for different stocks during two distinct time periods

\begin{tabular}{ccccccccc}
\hline \multicolumn{6}{c}{ Panel A: PIR for Hawkes implied beta relative to historical beta } \\
\hline Period & JPM & BAC & C & WFC & GS & MS & USB & COF \\
$2008-2009$ & 19.18 & 26.94 & 13.38 & 29.85 & 17.59 & 17.24 & 11.79 & 13.54 \\
$2016-2017$ & 16.86 & 5.49 & 6.52 & 8.92 & 8.95 & 6.19 & 8.11 & 8.08 \\
\hline \multicolumn{6}{c}{ Panel B: PIR for Poisson implied beta } & relative to historical beta \\
\hline Period & JPM & BAC & C & WFC & GS & MS & USB & COF \\
2008-2009 & 7.70 & 19.46 & 6.43 & 23.56 & 6.05 & 10.08 & 4.63 & 7.84 \\
$2016-2017$ & 6.09 & 1.77 & 1.94 & 4.85 & 3.94 & 4.32 & 4.18 & 2.83 \\
\hline Panel C: PIR for Hawkes implied beta relative to & Poisson implied beta \\
\hline Period & JPM & BAC & C & WFC & GS & MS & USB & COF \\
$2008-2009$ & 12.44 & 9.29 & 7.44 & 8.23 & 12.28 & 7.96 & 7.51 & 6.18 \\
$2016-2017$ & 11.47 & 3.79 & 4.68 & 4.28 & 5.22 & 1.96 & 4.11 & 5.40 \\
\hline \multicolumn{7}{c}{}
\end{tabular}

information tends to have superior predictive power over the historical information especially when the market is more volatile (c.f. French et al. 1983; Corrado and Su 1997; Chang et al. 2011). In addition, Panel $\mathrm{C}$ illustrates that, the prediction performance of the Hawkes implied beta relative to the Poisson implied beta is also much better in the financial crisis period than in the relatively steady period. It is consistent with the intuition that during a financial crisis, more significant jump clustering and jump spillover in stock prices will exist, and hence the option-implied beta of the Hawkes jump-diffusion model, which captures such clustered jump characteristics, will have better performance predicting the future beta in the financial crisis ${ }^{1}$. Given its importance for predicting the future beta, especially during a financial crisis period, the superior prediction ability of the implied beta under the Hawkes jump-diffusion model will enable investors to manage portfolios and systematic risk more effectively.

\section{Conclusion}

Significant market movements or a crisis typically occur with clustered jumps and often have spillover effects on other assets or markets as well. The occurrence of a jump in an equity price/return will increase the possibility, frequency, and magnitude of other equities due to different scales of dependence.

In this study, we extend the existing literature on exchange options by using a mutually exciting Hawkes jump-diffusion process to model the dynamics of two underlying assets. We develop a method to calculate the time-conditional joint characteristic function for the Hawkes jump-diffusion

\footnotetext{
${ }^{1}$ We also account for some other industries' representative companies and our results are robust. To conserve space, the results are not tabulated and are available upon request.
} 
process, from which we derive the specific pricing formula for the exchange option using a Fourier transform technique, and obtain the optimal hedging portfolio for the exchange option under the mean-variance criterion. We also present the Greeks and some other partial derivatives of the exchange option price under clustered jumps. Additionally, we perform a sensitivity analysis for the Hawkes-type jumps using numerical examples. We show the effects of the corresponding parameters in the two underlying assets on exchange option prices and how the parameters affect the exchange option price by contributing to the fluctuations of the assets. Moreover, we discuss the implications of incorporating clustered jumps in the exchange option-implied beta and compare the implied betas under different models. Using the proposed hybrid approach, we empirically shows that the prediction performance of the Hawkes implied beta are superior in comparison to the alternative estimates, especially during the financial crisis period. Our research provides insightful information for investors, risk managers, and researchers, and shows that exchange option models with clustered jumps can potentially provide substantial information.

In addition to pricing European-style exchange options with clustered price discontinuities, the proposed framework can be further extended to American-style exchange options or related derivatives, such as spread options, using numerical approaches. Future work could help extend the directional jumps techniques to further improve the forecasting capability of the implied beta and the dynamic hedging performance.

\section{Acknowledgements}

We would like to thank the two anonymous reviewers for their comments and suggestions. This research was supported by the National Natural Science Foundation of China (71601075, 71971077), and the Hunan Provincial Natural Science Foundation of China (2019JJ30001).

\section{References}

Aït-Sahalia, Y., Cacho-Diaz, J. and Laeven, R.J., Modeling financial contagion using mutually exciting jump processes. Journal of Financial Economics, 2015, 117, 585-606.

Amihud, Y., Lev, B. and Travlos, N.G., Corporate control and the choice of investment financing: The case of corporate acquisitions. The Journal of Finance, 1990, 45, 603-616.

Ané, T. and Métais, C., Are Jumps Contagious? An Empirical Investigation of Jumps Transmission Mechanisms in the Nasdaq Sector Indexes. Finance, 2011, 32, 11-41.

Antonelli, F., Ramponi, A. and Scarlatti, S., Exchange option pricing under stochastic volatility: a correlation expansion. Review of Derivatives Research, 2010, 13, 45-73.

Bacry, E., Delattre, S., Hoffmann, M. and Muzy, J.F., Modelling microstructure noise with mutually exciting point processes. Quantitative Finance, 2013, 13, 65-77.

Bacry, E. and Muzy, J.F., Hawkes model for price and trades high-frequency dynamics. Quantitative Finance, $2014, \mathbf{1 4}, 1147-1166$.

Bhagwat, V., Dam, R. and Harford, J., The real effects of uncertainty on merger activity. The Review of Financial Studies, 2016, 29, 3000-3034.

Biffis, E. and Millossovich, P., The fair value of guaranteed annuity options. Scandinavian Actuarial Journal, 2006, 2006, 23-41.

Bühlmann, H., Multidimensional valuation. Finance, 2004, 25, 15-29.

Buss, A. and Vilkov, G., Measuring equity risk with option-implied correlations. The Review of Financial Studies, 2012, 25, 3113-3140.

Butcher, J.C., The numerical analysis of ordinary differential equations: Runge-Kutta and general linear methods. , 1987.

Carr, P., The valuation of sequential exchange opportunities. The Journal of Finance, 1988, 43, 1235-1256.

Chang, B.Y., Christoffersen, P., Jacobs, K. and Vainberg, G., Option-implied measures of equity risk. Review of Finance, 2011, 16, 385-428. 
Cheang, G.H. and Chiarella, C., Exchange options under jump-diffusion dynamics. Applied Mathematical Finance, 2011, 18, 245-276.

Chen, X. and Wan, J., European Exchange Option Pricing in Exponential Lévy Model. In Proceedings of the Artificial Intelligence and Computational Intelligence (AICI), 2010 International Conference on, Vol. 1, pp. 83-87, 2010.

Corrado, C.J. and Su, T., Implied volatility skews and stock return skewness and kurtosis implied by stock option prices. The European Journal of Finance, 1997, 3, 73-85.

Cremers, K.M. and Petajisto, A., How active is your fund manager? A new measure that predicts performance. The Review of Financial Studies, 2009, 22, 3329-3365.

Cufaro Petroni, N. and Sabino, P., Pricing exchange options with correlated jump diffusion processes. Quantitative Finance, 2018, 18, 1-13.

Denis, D.J. and Macias, A.J., Material adverse change clauses and acquisition dynamics. Journal of Financial and Quantitative Analysis, 2013, 48, 819-847.

Duffie, D., Pan, J. and Singleton, K., Transform analysis and asset pricing for affine jump-diffusions. Econometrica, 2000, 68, 1343-1376.

Errais, E., Giesecke, K. and Goldberg, L.R., Affine point processes and portfolio credit risk. SIAM Journal on Financial Mathematics, 2006, 1, 642-665.

Fama, E.F. and French, K.R., Common risk factors in the returns on stocks and bonds. Journal of Financial Economics, 1993, 33, 3-56.

Fama, E.F. and MacBeth, J.D., Risk, return, and equilibrium: Empirical tests. Journal of Political Economy, 1973, 81, 607-636.

Frazzini, A., Friedman, J. and Pomorski, L., Deactivating active share. Financial Analysts Journal, 2016, 72, 14-21.

French, D.W., Groth, J.C. and Kolari, J.W., Current investor expectations and better betas. The Journal of Portfolio Management, 1983, 10, 12-17.

Gilson, R.J. and Schwartz, A., Understanding MACs: Moral hazard in acquisitions. Journal of Law, Economics, and Organization, 2005, 21, 330-358.

Hardy, M., Investment guarantees: modeling and risk management for equity-linked life insurance, Vol. 215, , 2003, John Wiley \& Sons.

Harris, R.D., Li, X. and Qiao, F., Option-implied betas and the cross section of stock returns. Journal of Futures Markets, 2019, 39, 94-108.

Hawkes, A.G., Spectra of some self-exciting and mutually exciting point processes. Biometrika, 1971, 58, $83-90$.

Hawkes, A.G., Hawkes processes and their applications to finance: A review. Quantitative Finance, 2018, 18, 193-198.

Herath, H.S. and Jahera Jr, J.S., Real options: valuing flexibility in strategic mergers and acquisitions as an exchange ratio swap. Managerial finance, 2002, 28, 44-62.

Johnson, S.A. and Tian, Y.S., Indexed executive stock options. Journal of Financial Economics, 2000, 57, 35-64.

Kardaras, C., Valuation and parities for exchange options. SIAM Journal on Financial Mathematics, 2015, 6, 140-157.

Kensinger, J.W., The capital investment project as a set of exchange options. Managerial Finance, 1988, 14, 16-27.

Kim, G., Kim, H. and Lee, S., Exchange option in a two-state Poisson CAPM. Journal of the Korean Statistical Society, 2013, 42, 507-513.

Kim, G. and Koo, E., Closed-form pricing formula for exchange option with credit risk. Chaos, Solitons 86 Fractals, 2016, 91, 221-227.

Kim, K.H., Sin, M.G. and Chong, U.H., Pricing formula for exchange option in fractional Black-Scholes model with jumps. Journal of Hyperstructures, 2014, 3, 155-164.

Kokholm, T., Pricing and hedging of derivatives in contagious markets. Journal of Banking EG Finance, 2016, 66, $19-34$.

Lewis, A., A simple option formula for general jump-diffusion and other exponential Lévy processes. Envision Financial Systems and OptionCity.net., 2001 Available at http://optioncity.net/pubs/ExpLevy.pdf.

Lin, L., Li, Y. and Wu, J., The pricing of European options on two underlying assets with delays. Physica A: Statistical Mechanics and its Applications, 2018, 495, 143-151.

Liu, C. and Wang, D.F., Exchange options and spread options with stochastic interest rates. University of 
Waterloo, Working Paper, 1999.

Luciano, E. and Schoutens, W., A multivariate jump-driven financial asset model. Quantitative Finance, 2006, 6, 385-402.

Ma, Y., Shrestha, K. and Xu, W., Pricing vulnerable options with jump clustering. Journal of Futures Markets, 2017, 37, 1155-1178.

Ma, Y. and $\mathrm{Xu}, \mathrm{W}$., Structural credit risk modelling with Hawkes jump diffusion processes. Journal of Computational \& Applied Mathematics, 2016, 303, 69-80.

Maheu, J.M. and McCurdy, T.H., News arrival, jump dynamics, and volatility components for individual stock returns. The Journal of Finance, 2004, 59, 755-793.

Margrabe, W., The value of an option to exchange one asset for another. The Journal of Finance, 1978, 33, $177-186$.

McNulty, J.J., Yeh, T.D., Schulze, W.S. and Lubatkin, M.H., What's your real cost of capital. Harvard Business Review, 2002, 80, 114-121.

Merton, R.C., Theory of rational option pricing. The Bell Journal of economics and management science, 1973, 31, 141-183.

Pablo, O. and Enrique, V., Valuing Exchange Options Under an Ornstein-Uhlenbeck Covariance Model. arXiv preprint arXiv:1711.10013, 2017.

Petajisto, A., Active share and mutual fund performance. Financial Analysts Journal, 2013, 69, 73-93.

Quittard-Pinon, F. and Randrianarivony, R., Exchange Options when One Underlying Price Can Jump. Finance, 2010, 31, 33-53.

Rubinstein, M., One for another. Risk, 1991, 4, 30-32.

Samuel, Y.M.Z.T., Option implied beta and option return. Applied Economics, 2018, 50, $128-142$.

Sharpe, W.F., Capital asset prices: A theory of market equilibrium under conditions of risk. The Journal of Finance, 1964, 19, 425-442.

Siegel, A.F., Measuring systematic risk using implicit beta. Management Science, 1995, 41, 124-128.

Trigeorgis, L., The nature of option interactions and the valuation of investments with multiple real options. Journal of Financial and Quantitative Analysis, 1993, 28, 1-20. 